\title{
Arithmetic of higher-dimensional orbifolds and a mixed Waring problem
}

\author{
Tim Browning $^{1} \cdot$ Shuntaro Yamagishi ${ }^{2}$
}

Received: 24 February 2019 / Accepted: 4 October 2020 / Published online: 5 March 2021

(c) The Author(s) 2021

\begin{abstract}
We study the density of rational points on a higher-dimensional orbifold $\left(\mathbb{P}^{n-1}, \Delta\right)$ when $\Delta$ is a $\mathbb{Q}$-divisor involving hyperplanes. This allows us to address a question of Tanimoto about whether the set of rational points on such an orbifold constitutes a thin set. Our approach relies on the Hardy-Littlewood circle method to first study an asymptotic version of Waring's problem for mixed powers. In doing so we make crucial use of the recent resolution of the main conjecture in Vinogradov's mean value theorem, due to Bourgain-Demeter-Guth and Wooley.
\end{abstract}

Mathematics Subject Classification 11D45 (11P55 · 14G05)

\section{Contents}

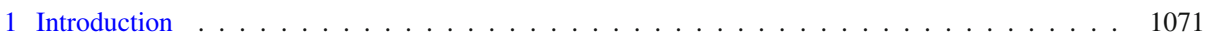

2 The Hardy-Littlewood circle method . . . . . . . . . . . . . . . . . . . . . . . . . . . 1077

3 Orbifold Manin: proof of Theorem 1.1 f . . . . . . . . . . . . . . . . . . . . . . . 1085

4 Thin sets: proof of Theorem $1.3 \ldots \ldots$. . . . . . . . . . . . . . . . . . . . . . . . . . . . . 1094

References . . . . . . . . . . . . . . . . . . . . . . . 1101

\section{Introduction}

This paper is about the arithmetic of rational points on higher-dimensional orbifolds, in the spirit of Campana [4]. We shall be concerned with orbifolds $\left(\mathbb{P}^{n-1}, \Delta\right)$, where $\Delta$ is a

Shuntaro Yamagishi

s.yamagishi@uu.nl

Tim Browning

tdb@ist.ac.at

1 IST Austria, Am Campus 1, 3400 Klosterneuburg, Austria

2 Mathematisch Instituut, Universiteit Utrecht, Budapestlaan 6, Utrecht NL 3584CD, The Netherlands 
$\mathbb{Q}$-divisor that takes the shape

$$
\Delta=\sum_{i=0}^{r}\left(1-\frac{1}{m_{i}}\right) D_{i}
$$

for irreducible divisors $D_{0}, \ldots, D_{r}$ on $\mathbb{P}^{n-1}$ and integers $m_{0}, \ldots, m_{r} \geq 2$. The arithmetic of Campana-points on orbifolds interpolates between the theory of rational and integral points on classical algebraic varieties, thereby opening up a new field of enquiry.

The orbifold $\left(\mathbb{P}^{n-1}, \Delta\right)$ is said to be smooth if the divisor $\sum_{i=0}^{r} D_{i}$ is strict normal crossings and it is said to be $\log$-Fano if $-K_{\mathbb{P}^{n-1}, \Delta}$ is ample, where $K_{\mathbb{P}^{n-1}, \Delta}=K_{\mathbb{P}^{n-1}}+\Delta$. Very recent work by Pieropan, Smeets, Tanimoto and Várilly-Alvarado [11] builds on the programme of Campana [4], by studying the distribution of Campana-points on vector group compactifications. Inspired by the Manin conjecture for rational points on Fano varieties [7], they formulate in [11, Conj. 1.1] a new prediction for the density of Campana-points of bounded height on arbitrary smooth log-Fano orbifolds.

We shall address this conjecture in the special case $\left(\mathbb{P}^{n-1}, \Delta\right)$, when the divisors $D_{0}, \ldots, D_{r}$ form a set of distinct hyperplanes in $\mathbb{P}^{n-1}$, all defined over $\mathbb{Q}$. Then $\left(\mathbb{P}^{n-1}, \Delta\right)$ is log-Fano precisely when

$$
n-(r+1)+\sum_{i=0}^{r} \frac{1}{m_{i}}>0 .
$$

Since $m_{i} \geq 2$ this forces us to have $r \leq 2(n-1)$. It turns out that the analysis is rather easy when $r \leq n-1$, a case that is covered as a special case of concurrent work by Pieropan and Schindler [10] on toric varieties. The first really challenging case is when $r=n$, in which case the condition for being log-Fano is

$$
\sum_{i=0}^{n} \frac{1}{m_{i}}>1
$$

We shall take

$$
D_{i}= \begin{cases}\left\{x_{i}=0\right\} & \text { if } 0 \leq i \leq n-1 \\ \left\{c_{0} x_{0}+\cdots+c_{n-1} x_{n-1}=0\right\} & \text { if } i=n\end{cases}
$$

for a fixed choice of non-zero integers $c_{0}, \ldots, c_{n-1}$. We let

$$
\Delta=\sum_{i=0}^{n}\left(1-\frac{1}{m_{i}}\right) D_{i}
$$

for given integers $m_{i} \geq 2$. The Campana-points in $\left(\mathbb{P}^{n-1}, \Delta\right)$ are defined to be the rational points $\left(x_{0}: \cdots: x_{n-1}\right) \in \mathbb{P}^{n-1}(\mathbb{Q})$, represented by primitive integer vectors $\left(x_{0}, \ldots, x_{n-1}\right) \in \mathbb{Z}_{\neq 0}^{n}$ for which $x_{i}$ is $m_{i}$-full for $0 \leq i \leq n-1$ and $c_{0} x_{0}+\cdots+c_{n-1} x_{n-1}$ is $m_{n}$-full. Here, we recall that a non-zero integer $x$ is said to be $m$-full if $p^{m} \mid x$ whenever there is a prime $p$ such that $p \mid x$.

We employ the height function $H: \mathbb{P}^{n-1}(\mathbb{Q}) \rightarrow \mathbb{R}$, given by

$$
H\left(x_{0}: \cdots: x_{n-1}\right)=\max _{0 \leq i \leq n-1}\left|x_{i}\right|,
$$


if $\left(x_{0}, \ldots, x_{n-1}\right) \in \mathbb{Z}^{n}$ is primitive. This is the standard exponential height associated to the line bundle $L=\mathscr{O}_{\mathbb{P}^{n-1}}(1)$. The counting function of interest to us here is then

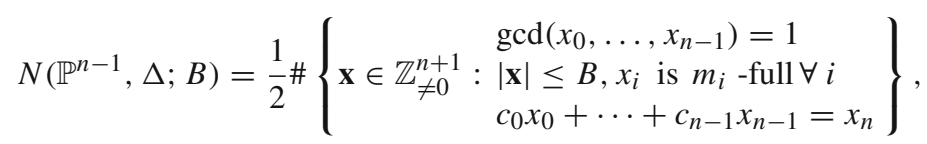

where $\mathbf{x}=\left(x_{0}, \ldots, x_{n}\right)$ and $|\mathbf{x}|=\max _{0 \leq i \leq n}\left|x_{i}\right|$. If $[L]$ denotes the divisor class of a hyperplane section in $\mathbb{P}^{n-1}$ and $\Lambda_{\text {eff }}$ is the effective cone of $\mathbb{P}^{n-1}$, then we define

$$
a=a(L, \Delta)=\inf \left\{t \in \mathbb{R}: t[L]+\left[K_{\mathbb{P}^{n-1}}+\Delta\right] \in \Lambda_{\mathrm{eff}}\right\} .
$$

Moreover, we define $b=b(L, \Delta)$ to be the codimension of the minimal supported face of $\Lambda_{\text {eff }}$ that contains the class $a[L]+\left[K_{\mathbb{P}^{n-1}}+\Delta\right]$. With these definitions in mind, the conjecture [11, Conj. 1.1] predicts that there exists a constant $c$ such that

$$
N\left(\mathbb{P}^{n-1}, \Delta ; B\right) \sim c B^{a}(\log B)^{b-1},
$$

as $B \rightarrow \infty$. (As a matter of fact, [11, Conj. 1.1] allows for the removal of a thin set of rational points from $\mathbb{P}^{n-1}(\mathbb{Q})$, a topic that we shall return to in our discussion of Theorem 1.2 below.) In our example, the degree function gives an isomorphism $\operatorname{Pic}\left(\mathbb{P}^{n-1}\right) \cong \mathbb{Z}$. Under this isomorphism $\Lambda_{\text {eff }} \cong \mathbb{R}_{\geq 0}$ and the line bundle $L$ has degree 1 . Moreover, $K_{\mathbb{P}^{n-1}}$ has degree $-n$ and $\Delta$ has degree $\sum_{i=0}^{n}\left(1-\frac{1}{m_{i}}\right)$. Hence

$$
a=\inf \left\{t \in \mathbb{R}: t+1-\sum_{i=0}^{n} \frac{1}{m_{i}} \geq 0\right\}=\sum_{i=0}^{n} \frac{1}{m_{i}}-1 .
$$

Furthermore, the minimal supported face of $\Lambda_{\text {eff }}$ which contains 0 is $\{0\}$, which has codimension 1 in $\Lambda_{\text {eff }}$, whence $b=1$.

In the special case $m_{0}=\cdots=m_{n}=2$, work of Van Valckenborgh [14] establishes an asymptotic formula for $N\left(\mathbb{P}^{n-1}, \Delta ; B\right)$ for all $n \geq 4$, which agrees with (1.2). Drawing inspiration from this, we have the following generalisation, which is also in accordance with (1.2).

Theorem 1.1 Assume that $m_{0}, \ldots, m_{n} \geq 2$ and

$$
\sum_{\substack{0 \leq i \leq n \\ i \neq j}} \frac{1}{m_{i}\left(m_{i}+1\right)} \geq 1
$$

for some $j \in\{0, \ldots, n\}$. Then there exist constants $c \geq 0$ and $\eta>0$ such that

$$
N\left(\mathbb{P}^{n-1}, \Delta ; B\right)=c B^{\sum_{i=0}^{n} \frac{1}{m_{i}}-1}+O\left(B^{\sum_{i=0}^{n} \frac{1}{m_{i}}-1-\eta}\right) .
$$

The implied constant in this estimate is allowed to depend on $m_{0}, \ldots, m_{n}, n$ and $c_{0}, \ldots, c_{n-1}$, a convention that we shall adopt for all of the implied constants in this paper. There is an explicit expression for the leading constant $c$ in (3.14) and (3.15), as a convergent sum of local densities. It can be shown that $c>0$ if the underlying equations admit suitable non-singular solutions everywhere locally.

Let us return briefly to the case $m_{0}=\cdots=m_{n}=2$, so that $a=\frac{n-1}{2}$ and $b=1$. When $n=3$ we have the following lower bound, in which the exponent of $\log B$ is at odds with the asymptotic formula (1.2). 
Theorem 1.2 Let $n=3$ and $m_{0}=m_{1}=m_{2}=m_{3}=2$. Then

$$
N\left(\mathbb{P}^{2}, \Delta ; B\right) \gg B \log B .
$$

As explained by Pieropan, Smeets, Tanimoto and Várilly-Alvarado [11, § 3.5], the points contributing to the lower bound for $N\left(\mathbb{P}^{2}, \Delta ; B\right)$ in this result actually correspond to rational points contained in a thin set in $\mathbb{P}^{2}(\mathbb{Q})$. Let $X$ be an integral variety over $\mathbb{Q}$. Recall from Serre $[13, \S 3.1]$ that a thin set is a set contained in a finite union of thin subsets of $X(\mathbb{Q})$ of type $I$ and $I I$. Here, a type $I$ thin subset is a set of the form $Z(\mathbb{Q}) \subset X(\mathbb{Q})$, where $Z$ is a proper closed subvariety, and a type II thin subset is a set of the form $f(Y(\mathbb{Q}))$, where $f: Y \rightarrow X$ is a generically finite dominant morphism with $\operatorname{dim} Y=\operatorname{dim} X, \operatorname{deg} f \geq 2$ and $Y$ geometrically integral. Theorem 1.2 illustrates that it is important to allow the possibility of removing thin sets of rational points from the statement of [11, Conj. 1.1].

On the other hand, when $m_{0}=m_{1}=m_{2}=2$ and $n=2$ we expect the counting function to satisfy an asymptotic formula of the form (1.2) with associated constants $a=\frac{1}{2}$ and $b=1$. In fact, Browning and Van Valckenborgh [2] have produced an explicit constant $c>0$ such that

$$
N\left(\mathbb{P}^{1}, \Delta ; B\right) \geq c(1+o(1)) B^{\frac{1}{2}}
$$

as $B \rightarrow \infty$.

A well-known result of Cohen [5], as expounded by Serre [12, Thm. 13.3], states that the set $\mathbb{P}^{n-1}(\mathbb{Q})$ is not thin. At the workshop "Rational and integral points via analytic and geometric methods" in Oaxaca (May 27th-June 1st, 2018), Sho Tanimoto raised the question of whether the same is true for the set of Campana-points. Our next goal is to provide some partial evidence in favour of this.

Associated to any type II thin subset $\Omega \subset \mathbb{P}^{n-1}(\mathbb{Q})$ coming from a morphism $Y \rightarrow \mathbb{P}^{n-1}$ of degree $d \geq 2$ is a degree $d$ extension of function fields $\mathbb{Q}(Y) / \mathbb{Q}\left(t_{1}, \ldots, t_{n-1}\right)$. We let $\mathbb{Q}(Y)^{\mathrm{Gal}}$ be the Galois closure of $\mathbb{Q}(Y)$ over the function field $\mathbb{Q}\left(t_{1}, \ldots, t_{n-1}\right)$ of $\mathbb{P}^{n-1}$ and we let $\mathbb{Q}_{\Omega} \subset \mathbb{Q}(Y)^{\mathrm{Gal}}$ be the largest subfield that is algebraic over $\mathbb{Q}$. Finally we let $\mathrm{P}_{\Omega}$ be the set of rational primes that split completely in $\mathbb{Q}_{\Omega}$. It follows from the Chebotarev density theorem that $P_{\Omega}$ has density $\left[\mathbb{Q}_{\Omega}: \mathbb{Q}\right]^{-1}$ in the set of primes, since $\mathbb{Q} \Omega / \mathbb{Q}$ is Galois. Next, let

$$
\mathbf{Q}_{\mathbf{m}}=\left\{p \text { prime : } \begin{array}{r}
\operatorname{lcm}\left(\operatorname{gcd}\left(m_{0}, p-1\right), \ldots, \operatorname{gcd}\left(m_{n}, p-1\right)\right) \\
=\prod_{0 \leq i \leq n} \operatorname{gcd}\left(m_{i}, p-1\right)
\end{array}\right\},
$$

for any $\mathbf{m}=\left(m_{0}, \ldots, m_{n}\right) \in \mathbb{Z}_{\geq 2}^{n+1}$. The following result provides an explicit condition on the possible thin sets in $\mathbb{P}^{n-1}(\mathbb{Q})$ that the Campana-points in $\left(\mathbb{P}^{n-1}, \Delta\right)$ are allowed to lie in.

Theorem 1.3 Assume that $m_{0}, \ldots, m_{n} \geq 2$ and (1.3) holds. Let $\Omega$ be a thin set contained in a finite union $\bigcup_{i} \Omega_{i}$, where each $\Omega_{i} \subset \mathbb{P}^{n-1}(\mathbb{Q})$ is a thin subset of type I or II. Assume that

$$
\liminf _{x \rightarrow \infty} \frac{\#\left\{p \in P_{\Omega_{i}} \cap Q_{\mathbf{m}}: p \leq x\right\}}{\pi(x)}>0
$$

whenever $\Omega_{i}$ is type II. Then

$$
N_{\Omega}\left(\mathbb{P}^{n-1}, \Delta ; B\right)=o_{\Omega}\left(B^{\sum_{i=0}^{n} \frac{1}{m_{i}}-1}\right),
$$

where $N_{\Omega}\left(\mathbb{P}^{n-1}, \Delta ; B\right)$ is defined as in (1.1), but with the additional constraint that the point $\left(x_{0}: \cdots: x_{n-1}\right) \in \Omega$. 
Assuming that (1.3) holds, we may combine this result with Theorem 1.1 to deduce that the Campana-points in $\left(\mathbb{P}^{n-1}, \Delta\right)$ are not contained in any thin subset of $\mathbb{P}^{n-1}(\mathbb{Q})$ satisfying the hypotheses of the theorem.

The statement of this result is rather disappointing at first glance, but in fact the conclusion is false when the condition (1.5) is dropped. To see this, take $m_{0}=\cdots=m_{n}=3$ and $n \geq 12$. Then $\sum_{i=0}^{n} \frac{1}{m_{i}}-1=\frac{n-2}{3}$ and (1.3) holds in Theorem 1.1. Consider the thin set $\Omega_{0} \subset \mathbb{P}^{n-1}(\mathbb{Q})$ that arises from the morphism

$$
Z \rightarrow \mathbb{P}^{n-1}, \quad\left(x_{0}: \cdots: x_{n}\right) \mapsto\left(x_{0}: \cdots: x_{n-1}\right),
$$

where $Z \subset \mathbb{P}^{n}$ is the cubic hypersurface $x_{0}^{3}+\cdots+x_{n-1}^{3}=x_{n}^{3}$. Then the counting function $N_{\Omega_{0}}\left(\mathbb{P}^{n-1}, \Delta ; B\right)$ has exact order $B^{\frac{n-2}{3}}$ for sufficiently large $n$. However, (1.5) fails in this case. Indeed, $\mathrm{Q}_{\mathbf{m}}$ is the set of primes $p \not \equiv 1 \bmod 3$, whereas $\mathrm{P}_{\Omega_{0}}$ is the set of primes $p \equiv 1 \bmod 3$, since $\mathbb{Q}_{\Omega_{0}}=\mathbb{Q}(\sqrt{-3})$. This shows that it is hard to approach Tanimoto's question in full generality through counting arguments alone.

The hypothesis (1.5) is a little awkward to work with. If one restricts attention to $\mathbf{m}$ such that

$$
\operatorname{gcd}\left(m_{j}, m_{j^{\prime}}\right)=1 \quad \text { for } 0 \leq j<j^{\prime} \leq n,
$$

then $\mathbf{Q}_{\mathbf{m}}$ is equal to the full set of rational primes. Moreover, it follows from the Chebotarev density theorem that $P_{\Omega}$ has density $\left[\mathbb{Q}_{\Omega}: \mathbb{Q}^{-1}\right.$, for any type II thin subset $\Omega$. Thus the conditions of Theorem 1.3 are met for any thin set. However, the assumption (1.3) is too stringent to cope with a sequence of integers $\geq 2$ that satisfies (1.6).

Our proof of Theorems 1.1-1.3 relies on an explicit description of $m$-full integers $x$. For such integers every exponent of a prime appearing in the prime factorisation of $x$ can be written $k m+(m+r)$, for integers $k \geq 0$ and $0 \leq r<m$. Thus any non-zero $m$-full integer $x$ can be written uniquely in the form

$$
x=\operatorname{sign}(x) u^{m} \prod_{r=1}^{m-1} v_{r}^{m+r},
$$

for $u, v_{1}, \ldots, v_{m-1} \in \mathbb{N}$, such that $\mu^{2}\left(v_{r}\right)=1$ for $1 \leq r \leq m-1$ and $\operatorname{gcd}\left(v_{r}, v_{r^{\prime}}\right)=1$ for $1 \leq r<r^{\prime} \leq m-1$.

It may be instructive to illustrate this notation by discussing the special case $m_{0}=\cdots=$ $m_{n}=2$, in which case Campana-points in $\left(\mathbb{P}^{n-1}, \Delta\right)$ correspond to vectors $\mathbf{u}, \mathbf{v} \in \mathbb{N}^{n+1}$ and $\boldsymbol{\epsilon} \in\{ \pm 1\}^{n+1}$ with each $v_{j}$ square-free, for which

$$
\epsilon_{0} c_{0} u_{0}^{2} v_{0}^{3}+\cdots+\epsilon_{n-1} c_{n-1} u_{n-1}^{2} v_{n-1}^{3}=\epsilon_{n} u_{n}^{2} v_{n}^{3} .
$$

When $n=3$ we can clearly find vectors $\mathbf{v} \in \mathbb{N}^{4}$ with square-free components and $\epsilon \in\{ \pm 1\}^{4}$ in such a way that

$$
-\epsilon_{0} \cdots \epsilon_{3} c_{0} c_{1} c_{2} v_{0}^{3} \cdots v_{3}^{3}=\square .
$$

Fixing such a choice and applying [8, Thm. 7] to estimate the residual number of $\mathbf{u} \in \mathbb{N}^{4}$ that lie on the split quadric, with $u_{j} \leq \sqrt{B / v_{j}^{3}}$ for $0 \leq j \leq 3$, we readily deduce that $N\left(\mathbb{P}^{2}, \Delta ; B\right) \gg B \log B$, as claimed in Theorem 1.2

Returning now to the case of general $m_{0}, \ldots, m_{n} \geq 2$, we summarise the structure of the paper. Under the representation (1.7) it follows that Campana-points on $\left(\mathbb{P}^{n-1}, \Delta\right)$ can be viewed through the lens of Waring's problem for mixed exponents. Given its proximity to 
Vinogradov's mean value theorem, this is an area that has received a radical new injection of ideas at the hands of Wooley [16,18,19] and Bourgain, Demeter and Guth [1]. Based on this, in Sect. 2 we shall give a completely general treatment of the counting function associated to suitably constrained integer solutions to the Diophantine equation

$$
\sum_{0 \leq j \leq n} c_{j} \gamma_{j} u_{j}^{m_{j}}=N
$$

for given $N \in \mathbb{Z}$ and non-zero $c_{j}, \gamma_{j} \in \mathbb{Z}$, in which the vectors $\mathbf{u}$ are asked to lie in a congruence class modulo $H$. In this part of the argument we shall need to retain uniformity in the coefficients $\gamma_{j}$ and in the modulus $H$. It is here that the condition (1.3) arises. The resulting asymptotic formula is recorded in Theorem 2.7. In Sect. 3 we shall use Theorem 2.7 to establish the version of orbifold Manin that we have presented in Theorem 1.1. One of the chief difficulties in this part of the argument comes from dealing with the coprimality conditions implicit in the counting function $N\left(\mathbb{P}^{n-1}, \Delta ; B\right)$. Next, in Sect. 4 we shall combine Theorem 2.7 with information about the size of thin sets modulo $p$ (for many primes $p$ ) to tackle Theorem 1.3.

Finally, when $H=1$ and $c_{j}=\gamma_{j}=1$ for all $0 \leq j \leq n$, it is easy to derive from Theorem 2.7 an asymptotic formula for the mixed Waring problem. The following result may be of independent interest.

Theorem 1.4 Assume that $m_{0}, \ldots, m_{n} \geq 2$ and (1.3) holds. Let $R(N)$ denote the number of representations of a positive integer $N$ as

$$
N=x_{0}^{m_{0}}+\cdots+x_{n}^{m_{n}} .
$$

Then there exists $\eta>0$ such that

$$
R(N)=\frac{\prod_{i=0}^{n} \Gamma\left(1+\frac{1}{m_{i}}\right)}{\Gamma\left(\sum_{i=0}^{n} \frac{1}{m_{i}}\right)} \mathfrak{S}(N) N^{\sum_{i=0}^{n} \frac{1}{m_{i}}-1}+O\left(N^{\sum_{i=0}^{n} \frac{1}{m_{i}}-1-\eta}\right),
$$

where $\mathfrak{S}(N)$ is given by (2.19).

There is relatively little in the literature concerning asymptotic formulae for $R(N)$ for mixed exponents. The best result is due to Brüdern [3] who obtains an asymptotic formula for $R(N)$ when $m_{0}=m_{1}=2$, under some further conditions on the exponents, the most demanding of which is that

$$
\sum_{i=2}^{n} \frac{1}{m_{i}}>1
$$

Theorem 1.4 is not competitive with this, although it does not suffer from the defect that 2 must appear twice among the list of exponents. It remains an interesting open challenge to prove an asymptotic formula for $R(N)$ for any value of $n$, when $m_{i}=2+i$ for $0 \leq i \leq n$.

When $m=m_{0}=\cdots=m_{n}$, which is the traditional setting of Waring's problem, the condition in (1.3) reduces to $n \geq m^{2}+m$. This shows that our approach is not completely optimal in the equal exponent situation, since as explained in [19, Cor. 14.7], we know that $n \geq m^{2}-m+O(\sqrt{m})$ variables suffice to get an asymptotic formula in Waring's problem. It seems likely that by combining methods developed by Wooley in [17] and [19, §14], one can recover this loss. (The authors are grateful to Professor Wooley for this remark.) 


\section{The Hardy-Littlewood circle method}

We shall assume without loss of generality that $2 \leq m_{0} \leq m_{1} \leq \cdots \leq m_{n}$. Our assumption (1.3) translates into

$$
\sum_{0 \leq j<n} \frac{1}{m_{j}\left(m_{j}+1\right)} \geq 1 .
$$

In what follows it will be convenient to set

$$
\Gamma=\sum_{j=0}^{n} \frac{1}{m_{j}}-1 .
$$

Let $N \in \mathbb{Z}$ and let $\mathbf{c}=\left(c_{0}, \ldots, c_{n}\right) \in(\mathbb{Z} \backslash\{0\})^{n+1}$. Let $H \in \mathbb{N}, \boldsymbol{\gamma} \in \mathbb{N}^{n+1}$ and let $\mathbf{h} \in$ $\{0,1, \ldots, H-1\}^{n+1}$. The main results in this paper are founded on an analysis of the counting function

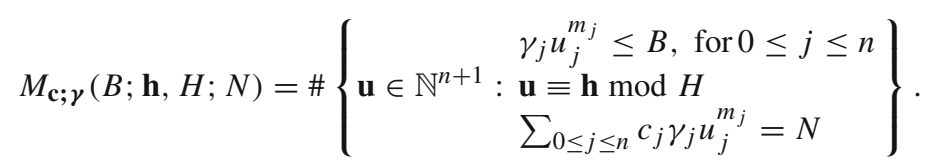

We shall view $\mathbf{c}$ as being fixed, once and for all, but $\boldsymbol{\gamma}$ can grow and so we will need all of our estimates to depend explicitly on it. In Theorems 1.1 and 1.3 we shall take $N=0$ and $c_{n}=-1$, whereas in Theorem 1.4 we take $H=1, c_{j}=\gamma_{j}=1$ and $B=N$.

We let $e(z)=\exp (2 \pi i z)$ for any $z \in \mathbb{R}$. Let

$$
B_{j}=\left(B / \gamma_{j}\right)^{1 / m_{j}}
$$

and

$$
S_{j}(\alpha)=\sum_{\substack{1 \leq u \leq B_{j} \\ u \equiv h_{j} \bmod H}} e\left(\alpha c_{j} \gamma_{j} u^{m_{j}}\right),
$$

for $0 \leq j \leq n$. Then we may write

$$
M_{\mathbf{c} ; \gamma}(B ; \mathbf{h}, H ; N)=\int_{0}^{1} \mathscr{S}_{\gamma}(\alpha) \mathrm{d} \alpha,
$$

where

$$
\mathscr{S}_{\gamma}(\alpha)=e(-\alpha N) \prod_{j=0}^{n} S_{j}(\alpha) .
$$

Note that we may freely assume that $\gamma_{j} \leq B$ for $0 \leq j \leq n$, since otherwise $M_{\mathbf{c} ; \gamma}(B ; \mathbf{h}, H ; N)=0$. Let $\delta$ be such that

$$
0<\delta<\frac{1}{(2 n+5) m_{n}\left(m_{n}+1\right)} .
$$

We define the major arcs $\mathfrak{M}$ to be

$$
\mathfrak{M}=\bigcup_{\substack{0 \leq a \leq q \leq B^{\delta} \\ \operatorname{gcd}(a, q)=1}} \mathfrak{M}(a, q),
$$


where

$$
\mathfrak{M}(a, q)=\left\{\alpha \in[0,1):|\alpha-a / q|<B^{-1+\delta}\right\} .
$$

We define the minor arcs to be $\mathfrak{m}=[0,1) \backslash \mathfrak{M}$.

\subsection{Contribution from the major arcs}

In the standard way we shall need to show that on the major arcs our exponential sums can be approximated by integrals, with acceptable error. The following result is a straightforward adaptation of familiar facts.

Lemma 2.1 Let $h, H \in \mathbb{N} \cup\{0\}$ with $0 \leq h<H$. Let $X \geq 1$. Let $a \in \mathbb{Z}, q \in \mathbb{N}, \beta \in \mathbb{R}$ and $\alpha=a / q+\beta$. Then

$$
\begin{gathered}
\sum_{\substack{1 \leq u \leq X \\
u \equiv h \bmod H}} e\left(\alpha u^{m}\right)=\frac{X}{q H} \sum_{k=0}^{q-1} e\left(\frac{a(H k+h)^{m}}{q}\right) \int_{0}^{1} e\left(\beta X^{m} z^{m}\right) \mathrm{d} z \\
+O\left(q+q X^{m}|\beta|\right) .
\end{gathered}
$$

Proof Let $X^{\prime}=(X-h) / H$. If $X^{\prime}<q$ then the absolute value of the left hand side is trivially bounded by $q+1$, and so we may proceed under the assumption that $X^{\prime} \geq q$. We write

$$
\begin{aligned}
\sum_{\substack{1 \leq u \leq X \\
u \equiv h \bmod H}} e\left(\alpha u^{m}\right) & =\sum_{0<x \leq X^{\prime}} e\left(\alpha(H x+h)^{m}\right)+O(1) \\
& =\sum_{k=0}^{q-1} e\left(\frac{a(H k+h)^{m}}{q}\right) \sum_{\substack{0<x \leq X^{\prime} \\
x \equiv k \bmod q}} e\left(\beta(H x+h)^{m}\right)+O(1) .
\end{aligned}
$$

The inner sum is

$$
\sum_{\substack{0<x \leq X^{\prime} \\ x \equiv k \bmod q}} e\left(\beta(H x+h)^{m}\right)=\sum_{-k / q<y \leq\left(X^{\prime}-k\right) / q} e\left(\beta(q H y+h+H k)^{m}\right) .
$$

An application of the Euler-Maclaurin summation formula to this sum now yields the result.

Now let $\alpha=a / q+\beta \in \mathfrak{M}(a, q)$. We apply Lemma 2.1 with $X=B_{j}$, and $\alpha$ (resp. $a$ ) replaced by $\alpha c_{j} \gamma_{j}$ (resp. $a c_{j} \gamma_{j}$ ). Thus $\alpha c_{j} \gamma_{j}-a c_{j} \gamma_{j} / q=\beta c_{j} \gamma_{j}$ and

$$
q+q B_{j}^{m_{j}}\left|\beta c_{j} \gamma_{j}\right| \ll q+q B|\beta| \ll B^{2 \delta} .
$$

Put

$$
\mathfrak{J}_{\mathbf{c}}(L)=\int_{-L}^{L} e(-\lambda N / B) \prod_{j=0}^{n} \int_{0}^{1} e\left(\lambda c_{j} z^{m_{j}}\right) \mathrm{d} z \mathrm{~d} \lambda
$$

and set $\mathfrak{S}_{\mathbf{c} ; \gamma}(L ; \mathbf{h}, H ; N)$ to be

$$
\sum_{q \leq L} \frac{1}{q^{n+1}} \sum_{\substack{0 \leq a<q \\ \operatorname{gcd}(a, q)=1}} e\left(-\frac{a N}{q}\right) \prod_{j=0}^{n} \sum_{0 \leq k<q} e\left(\frac{a}{q} c_{j} \gamma_{j}\left(H k+h_{j}\right)^{m_{j}}\right),
$$


for any $L>1$. Then it follows from Lemma 2.1 that

$$
\int_{\mathfrak{M}} \mathscr{S}_{\gamma}(\alpha) \mathrm{d} \alpha=\mathfrak{S}_{\mathbf{c} ; \boldsymbol{\gamma}}\left(B^{\delta} ; \mathbf{h}, H ; N\right) \mathfrak{J}_{\mathbf{c}}\left(B^{\delta}\right) \frac{\prod_{j=0}^{n} B_{j}}{H^{n+1} B}+O\left(E_{1}(\boldsymbol{\gamma} ; H)\right),
$$

where

$$
E_{1}(\boldsymbol{\gamma} ; H)=B^{-1+\delta} \sum_{q \leq B^{\delta}} q \sum_{y=0}^{n}\left(B^{2 \delta}\right)^{n+1-y} \max _{j_{1}<\cdots<j_{y}} \prod_{i=1}^{y}\left(\frac{B_{j_{i}}}{H}+1\right) .
$$

Taking $H \geq 1$ and observing that $B_{j} \geq 1$ for all $0 \leq j \leq n$ we see that

$$
\max _{j_{1}<\cdots<j_{y}} \prod_{i=1}^{y}\left(\frac{B_{j_{i}}}{H}+1\right) \ll\left(\frac{1}{B_{0}}+\cdots+\frac{1}{B_{n}}\right) \prod_{j=0}^{n} B_{j} .
$$

On executing the sum over $q$ we therefore conclude that

$$
E_{1}(\gamma ; H) \ll \frac{\prod_{j=0}^{n} B_{j}}{B}\left(\frac{1}{B_{0}}+\cdots+\frac{1}{B_{n}}\right) B^{(2 n+5) \delta} .
$$

It remains to analyse the terms $\mathfrak{S}_{\mathbf{c} ; \boldsymbol{\gamma}}\left(B^{\delta} ; \mathbf{h}, H ; N\right)$ and $\mathfrak{J}_{\mathbf{c}}\left(B^{\delta}\right)$.

Beginning with the singular series, it follows from [15, Theorem 7.1] that

$$
\left|\sum_{0 \leq k<q} e\left(\frac{x(H k+h)^{m}}{q}\right)\right| \ll \operatorname{gcd}(x, q)^{1 / m} H q^{1-1 / m+\varepsilon}
$$

for any $\varepsilon>0$. Therefore

$$
\begin{gathered}
\left|\sum_{X<q \leq Y} \frac{1}{q^{n+1}} \sum_{\substack{0 \leq a<q \\
\operatorname{gcd}(a, q)=1}} e\left(-\frac{a N}{q}\right) \prod_{j=0}^{n} \sum_{0 \leq k<q} e\left(\frac{a}{q} c_{j} \gamma_{j}\left(H k+h_{j}\right)^{m_{j}}\right)\right| \\
\ll E_{2}(\boldsymbol{\gamma} ; H ; X, Y),
\end{gathered}
$$

where

$$
E_{2}(\boldsymbol{\gamma} ; H ; X, Y)=H^{n+1} \sum_{X<q \leq Y} q^{-\Gamma+\varepsilon} \prod_{j=0}^{n} \operatorname{gcd}\left(\gamma_{j}, q\right)^{1 / m_{j}}
$$

Put

$$
E_{2}(\boldsymbol{\gamma} ; H)=H^{n+1} \sum_{q=1}^{\infty} q^{1-\Gamma+\varepsilon} \prod_{j=0}^{n} \operatorname{gcd}\left(\gamma_{j}, q\right)^{1 / m_{j}}
$$

Clearly $E_{2}\left(\boldsymbol{\gamma} ; H ; B^{\delta}, \infty\right) \leq B^{-\delta} E_{2}(\boldsymbol{\gamma} ; H)$ and $E_{2}(\boldsymbol{\gamma} ; H ; 0, \infty) \leq E_{2}(\boldsymbol{\gamma} ; H)$.

In view of (2.1), we have

$$
\sum_{j=0}^{n} \frac{1}{m_{j}}>3
$$


Let us define

$$
\begin{aligned}
\mathfrak{S}_{\mathbf{c} ; \gamma}(\mathbf{h}, H ; N)=\sum_{q=1}^{\infty} \frac{1}{q^{n+1}} & \sum_{\substack{0 \leq a<q \\
\operatorname{gcd}(a, q)=1}} e\left(-\frac{a N}{q}\right) \\
& \times \prod_{j=0}^{n} \sum_{0 \leq k<q} e\left(\frac{a}{q} c_{j} \gamma_{j}\left(H k+h_{j}\right)^{m_{j}}\right) .
\end{aligned}
$$

This is absolutely convergent, since (2.10) yields

$$
\mathfrak{S}_{\mathbf{c} ; \boldsymbol{\gamma}}(\mathbf{h}, H ; N) \ll E_{2}(\boldsymbol{\gamma} ; H ; 0, \infty) \ll E_{2}(\boldsymbol{\gamma} ; H) \ll H^{n+1} \prod_{j=0}^{n} \gamma_{j}^{1 / m_{j}} .
$$

Moreover,

$$
\mathfrak{S}_{\mathbf{c} ; \gamma}\left(B^{\delta} ; \mathbf{h}, H ; N\right)=\mathfrak{S}_{\mathbf{c} ; \boldsymbol{\gamma}}(\mathbf{h}, H ; N)+O\left(B^{-\delta} E_{2}(\boldsymbol{\gamma} ; H)\right) .
$$

Turning to the singular integral, it follows from [15, Lemma 2.8] that

$$
\int_{0}^{1} e\left(\lambda c_{j} z^{m_{j}}\right) \mathrm{d} z \ll \min \left\{1,|\lambda|^{-1 / m_{j}}\right\} .
$$

Thus, in view of (2.10), we deduce that

$$
\int_{|\lambda| \geq B^{\delta}} \prod_{j=0}^{n}\left|\int_{0}^{1} e\left(\lambda c_{j} z^{m_{j}}\right) \mathrm{d} z\right| \mathrm{d} \lambda \ll \int_{|\lambda| \geq B^{\delta}}|\lambda|^{-\sum_{j=0}^{n} \frac{1}{m_{j}}} \mathrm{~d} \lambda \ll B^{-\delta \Gamma} .
$$

Hence

$$
\mathfrak{J}_{\mathbf{c}}=\int_{-\infty}^{\infty} e(-\lambda N / B) \prod_{j=0}^{n} \int_{0}^{1} e\left(\lambda c_{j} z^{m_{j}}\right) \mathrm{d} z \mathrm{~d} \lambda
$$

is well-defined, and we have

$$
\left|\mathfrak{J}_{\mathbf{c}}-\mathfrak{J}_{\mathbf{c}}\left(B^{\delta}\right)\right| \ll B^{-\delta \Gamma} \leq B^{-\delta} .
$$

We are now ready to conclude our treatment of the major arcs. Note that

$$
\frac{\prod_{j=0}^{n} B_{j}}{H^{n+1} B}=\frac{B^{\Gamma}}{H^{n+1} \prod_{j=0}^{n} \gamma_{j}^{1 / m_{j}}} .
$$

On combining (2.6), (2.12), (2.13) and (2.14), we therefore obtain the following result.

Lemma 2.2 Assume that (2.10) holds. Then

$$
\int_{\mathfrak{M}} \mathscr{S}_{\boldsymbol{\gamma}}(\alpha) \mathrm{d} \alpha=\frac{\mathfrak{S}_{\mathbf{c} ; \boldsymbol{\gamma}}(\mathbf{h}, H ; N) \mathfrak{J}_{\mathbf{c}}}{H^{n+1} \prod_{j=0}^{n} \gamma_{j}^{1 / m_{j}}} B^{\Gamma}+O\left(E_{1}(\boldsymbol{\gamma} ; H)+\frac{B^{\Gamma-\delta} E_{2}(\boldsymbol{\gamma} ; H)}{H^{n+1} \prod_{j=0}^{n} \gamma_{j}^{1 / m_{j}}}\right) .
$$




\subsection{Contribution from the minor arcs}

According to work of Wooley [19, Eq. (1.8)], the main conjecture in Vinogradov's mean value theorem asserts that for each $\varepsilon>0$ and $t, k \in \mathbb{N}$, one has

$$
\int_{[0,1)^{k}}\left|\sum_{1 \leq x \leq X} e\left(\alpha_{k} x^{k}+\alpha_{k-1} x^{k-1}+\cdots+\alpha_{1} x\right)\right|^{2 t} \mathrm{~d} \boldsymbol{\alpha} \ll X^{t+\varepsilon}+X^{2 t-\frac{k(k+1)}{2}} .
$$

This result was established recently by Bourgain, Demeter and Guth [1] using $\ell^{2}$-decoupling and also by Wooley $[18,19]$ using efficient congruencing. The following mean value estimate is a straightforward consequence of their work.

Lemma 2.3 Let $k \in \mathbb{N}$ and let $s$ be a real number satisfying $s \geq k(k+1)$. Let $A, H \in \mathbb{Z} \backslash\{0\}$ and $h \in \mathbb{Z}$. Then we have

$$
\int_{0}^{1}\left|\sum_{1 \leq x \leq X} e\left(\alpha A(H x+h)^{k}\right)\right|^{s} \mathrm{~d} \alpha \ll X^{s-k},
$$

where the implied constant does not depend on A, $H$ or $h$.

Proof Let $2 t$ be the largest even integer such that $2 t \leq s$. Then it follows that $t \geq k(k+1) / 2$. Hence

$$
\int_{0}^{1}\left|\sum_{1 \leq x \leq X} e\left(\alpha A(H x+h)^{k}\right)\right|^{s} \mathrm{~d} \alpha \leq X^{s-2 t} \int_{0}^{1}\left|\sum_{1 \leq x \leq X} e\left(\alpha A(H x+h)^{k}\right)\right|^{2 t} \mathrm{~d} \alpha .
$$

On considering the underlying equations of the integral on the right hand side using the orthogonality relation, we deduce that the integral on the right hand side is the number of

$$
1 \leq x_{1}, \ldots, x_{t}, y_{1}, \ldots, y_{t} \leq X
$$

such that

$$
\sum_{i=1}^{t} A\left(H x_{i}+h\right)^{k}=\sum_{i=1}^{t} A\left(H y_{i}+h\right)^{k}
$$

It therefore follows that

$$
\int_{0}^{1}\left|\sum_{1 \leq x \leq X} e\left(\alpha A(H x+h)^{k}\right)\right|^{s} \mathrm{~d} \alpha \leq X^{s-2 t} \sum_{\substack{\mathbf{n}=\left(n_{1}, \ldots, n_{k-1}\right) \in \mathbb{Z}^{k-1} \\-t X^{j}<n_{j}<t X^{j}}} I(\mathbf{n}),
$$

where $I(\mathbf{n})$ is the number of (2.16) satisfying (2.17), for which

$$
\sum_{i=1}^{t} x_{i}^{j}-\sum_{i=1}^{t} y_{i}^{j}=n_{j}
$$

for $1 \leq j<k$. We may clearly write

$$
I(\mathbf{n})=\int_{[0,1)^{k}}\left|\sum_{1 \leq x \leq X} e\left(\alpha_{k} A(H x+h)^{k}+\alpha_{k-1} x^{k-1}+\cdots+\alpha_{1} x\right)\right|^{2 t} e\left(-\mathbf{n} \cdot \boldsymbol{\alpha}^{\prime}\right) \mathrm{d} \boldsymbol{\alpha},
$$


where $\boldsymbol{\alpha}=\left(\alpha_{k}, \ldots, \alpha_{1}\right)$ and $\boldsymbol{\alpha}^{\prime}=\left(\alpha_{k-1}, \ldots, \alpha_{1}\right)$. Summing trivially over $\mathbf{n}$, the right hand side of our estimate is

$$
\begin{aligned}
& \ll X^{\frac{k(k-1)}{2}} \int_{[0,1)^{k}}\left|\sum_{1 \leq x \leq X} e\left(\alpha_{k} A(H x+h)^{k}+\alpha_{k-1} x^{k-1}+\cdots+\alpha_{1} x\right)\right|^{2 t} \mathrm{~d} \boldsymbol{\alpha} \\
& =X^{\frac{k(k-1)}{2}} \int_{[0,1)^{k}}\left|\sum_{1 \leq x \leq X} e\left(\alpha_{k} x^{k}+\alpha_{k-1} x^{k-1}+\cdots+\alpha_{1} x\right)\right|^{2 t} \mathrm{~d} \boldsymbol{\alpha},
\end{aligned}
$$

where the last equality is an immediate consequence of the fact that

$$
I(\mathbf{0})=\#\left\{1 \leq x_{1}, \ldots, x_{t}, y_{1}, \ldots, y_{t} \leq X: \sum_{i=1}^{t} x_{i}^{j}=\sum_{i=1}^{t} y_{i}^{j}(1 \leq j \leq k)\right\} .
$$

An application of (2.15) now yields our result.

We also require the following Weyl type estimate, which is another consequence of the recent work on Vinogradov's mean value theorem. We omit the proof since it is obtained by invoking the main conjecture (2.15) in the proof of [16, Theorem 1.5].

Lemma 2.4 Let $k \geq 2$ and let $\alpha_{k}, \ldots, \alpha_{1} \in \mathbb{R}$. Suppose there exist $a \in \mathbb{Z}$ and $q \in \mathbb{N}$ with $\operatorname{gcd}(a, q)=1$ satisfying $\left|\alpha_{k}-a / q\right| \leq q^{-2}$ and $q \leq X^{k}$. Let

$$
0 \leq \sigma \leq \frac{1}{k(k-1)} \text {. }
$$

Then

$$
\sum_{1 \leq x \leq X} e\left(\alpha_{k} x^{k}+\alpha_{k-1} x^{k-1}+\cdots+\alpha_{1} x\right) \ll X^{1+\varepsilon}\left(q^{-1}+X^{-1}+q X^{-k}\right)^{\sigma},
$$

for any $\varepsilon>0$.

Using this result we obtain the following bound for the exponential sum on the minor arcs.

Lemma 2.5 Let $\varepsilon>0$. Then

$$
\sup _{\alpha \in \mathfrak{m}}\left|S_{n}(\alpha)\right| \ll B^{\frac{1}{m_{n}}-\frac{\delta}{m_{n}\left(m_{n}+1\right)}+\varepsilon} \gamma_{n}^{-\frac{1}{m_{n}+1}} .
$$

Proof It will be convenient throughout the proof to write

$$
\sigma\left(m_{n}\right)=\frac{1}{m_{n}\left(m_{n}+1\right)} .
$$

Let $\alpha \in \mathfrak{m}$ and let $\beta=\alpha c_{n} \gamma_{n} H^{m_{n}}$. We put

$$
\widetilde{B}=\min \left\{2 B^{1-\delta}, \frac{B}{\left|c_{n}\right| \gamma_{n} H^{m_{n}} 2^{m_{n}}}\right\} .
$$

When $\widetilde{B} \leq 1$ it is clear that $S_{n}(\alpha) \ll 1$. Since $\gamma_{n} \leq B$ we have

$$
B^{1 / m_{n}-\delta \sigma\left(m_{n}\right)+\varepsilon} \gamma_{n}^{-1 / m_{n}+\sigma\left(m_{n}\right)} \geq 1 \gg S_{n}(\alpha)
$$

in this case. Thus we may suppose that $\widetilde{B}>1$. 
By Dirichlet's theorem on Diophantine approximation we know there exist $b \in \mathbb{Z}$ and $1 \leq r \leq \widetilde{B}$ such that $\operatorname{gcd}(b, r)=1$ and

$$
|\beta-b / r| \leq 1 /(r \widetilde{B}) \leq 1 / r^{2} .
$$

Note that $b \neq 0$ since $\alpha \in \mathfrak{m}$. For simplicity let us write $A=c_{n} \gamma_{n} H^{m_{n}}$. We claim that $b A>0$. But if $b A<0$ then

$$
|\beta-b / r|=|\alpha A-b / r|=\alpha|A|+|b| / r>1 / r,
$$

since $\alpha>0$, which is a contradiction. This establishes the claim. Let $A^{\prime}=A / \operatorname{gcd}(A, b)$ and $b^{\prime}=b / \operatorname{gcd}(A, b)$.

Let $X=\left(B_{n}-h_{n}\right) / H$. First suppose $B_{n} / 2 H>X$. Then $B_{n}<2 h_{n}<2 H$. In this case we clearly have $S_{n}(\alpha) \ll 1$, which is satisfactory. Thus we suppose $B_{n} / 2 H \leq X$. In this case $r \leq \widetilde{B} \leq X^{m_{n}}$ and Lemma 2.4 yields

$$
\begin{aligned}
S_{n}(\alpha) & =\sum_{1 \leq x \leq X} e\left(\beta\left(x+\frac{h}{H}\right)^{m_{n}}\right)+O(1) \\
& \ll X^{1+\varepsilon}\left(r^{-1}+X^{-1}+r X^{-m_{n}}\right)^{\sigma\left(m_{n}\right)},
\end{aligned}
$$

for any $\varepsilon>0$. Next, we note that

$$
\frac{1}{|A| r \widetilde{B}} \geq \frac{1}{|A|}\left|\beta-\frac{b}{r}\right|=\left|\alpha-\frac{b}{r A}\right|=\left|\alpha-\frac{\left|b^{\prime}\right|}{r\left|A^{\prime}\right|}\right| .
$$

If $2 B^{1-\delta} \leq B /\left|c_{n} \gamma_{n} H^{m_{n}} 2^{m_{n}}\right|$ it follows that

$$
\left|\alpha-\frac{\left|b^{\prime}\right|}{r\left|A^{\prime}\right|}\right| \leq \frac{1}{|A| r \widetilde{B}} \leq \frac{1}{\widetilde{B}}<B^{-1+\delta} .
$$

On the other hand, if $2 B^{1-\delta}>B /\left|c_{n} \gamma_{n} H^{m_{n}} 2^{m_{n}}\right|$ then

$$
\left|\alpha-\frac{\left|b^{\prime}\right|}{r\left|A^{\prime}\right|}\right| \leq \frac{1}{|A| r \widetilde{B}} \leq \frac{\left|c_{n}\right| \gamma_{n} H^{m_{n}} 2^{m_{n}}}{|A| B} \ll \frac{1}{B} .
$$

We now verify that $1 \leq\left|b^{\prime}\right| \leq r\left|A^{\prime}\right|$. We've already seen that $\left|b^{\prime}\right| \geq 1$, so we suppose that $\left|b^{\prime}\right|>r\left|A^{\prime}\right|$. Since $\alpha \in[0,1)$ we have

$$
\frac{1}{r\left|A^{\prime}\right|} \leq\left|\alpha-\frac{\left|b^{\prime}\right|}{r\left|A^{\prime}\right|}\right| \leq \frac{1}{|A| r \widetilde{B}}
$$

whence $1<\widetilde{B} \leq\left|A^{\prime}\right| /|A| \leq 1$. This is a contradiction, so that we do indeed have $1 \leq\left|b^{\prime}\right| \leq$ $r\left|A^{\prime}\right|$. We also have $\operatorname{gcd}\left(r\left|A^{\prime}\right|,\left|b^{\prime}\right|\right)=1$. Finally, $\alpha \in \mathfrak{M}$ if $r\left|A^{\prime}\right| \leq B^{\delta}$ and $B$ exceeds an appropriate constant. But this is a contradiction, so that $r\left|A^{\prime}\right|>B^{\delta}$ and (2.18) becomes

$$
\begin{aligned}
S_{n}(\alpha) & \ll \frac{B^{1 / m_{n}+\varepsilon}}{|A|^{1 / m_{n}}}\left(\left|A^{\prime}\right| / B^{\delta}+(B /|A|)^{-1 / m_{n}}+|A| / B^{\delta}\right)^{\sigma\left(m_{n}\right)} \\
& \ll \frac{B^{1 / m_{n}+\varepsilon}}{|A|^{1 / m_{n}}}\left((B /|A|)^{-1 / m_{n}}+|A| / B^{\delta}\right)^{\sigma\left(m_{n}\right)} \\
& \ll B^{1 / m_{n}-\delta \sigma\left(m_{n}\right)+\varepsilon}|A|^{\sigma\left(m_{n}\right)-1 / m_{n}} .
\end{aligned}
$$

This completes the proof of the lemma, since $\sigma\left(m_{n}\right)-1 / m_{n}=-1 /\left(m_{n}+1\right)$.

We now have the tools in place to establish the following bound for the minor arc contribiution. 
Lemma 2.6 Assume that (2.1) holds and let $\varepsilon>0$. Then

$$
\int_{\mathfrak{m}}\left|\prod_{j=0}^{n} S_{j}(\alpha)\right| \mathrm{d} \alpha \ll B^{\Gamma-\frac{\delta}{m_{n}\left(m_{n}+1\right)}}+\varepsilon \prod_{j=0}^{n} \gamma_{j}^{-\frac{1}{m_{j}+1}}
$$

Proof Let $\ell_{n}=m_{n}\left(m_{n}+1\right)$ and let $\ell_{0}, \cdots, \ell_{n-1}>0$ be such that

$$
\sum_{0 \leq j<n} \frac{1}{\ell_{j}}=1
$$

In the light of (2.1) we can assume that $\ell_{j} \geq m_{j}\left(m_{j}+1\right)$ for all $0 \leq j \leq n-1$. It now follows from Hölder's inequality and Lemma 2.3 that

$$
\begin{aligned}
\int_{\mathfrak{m}}\left|\prod_{j=0}^{n} S_{j}(\alpha)\right| \mathrm{d} \alpha & \leq \sup _{\alpha \in \mathfrak{m}}\left|S_{n}(\alpha)\right| \cdot \int_{0}^{1}\left|\prod_{j=0}^{n-1} S_{j}(\alpha)\right| \mathrm{d} \alpha \\
& \leq \sup _{\alpha \in \mathfrak{m}}\left|S_{n}(\alpha)\right| \cdot \prod_{j=0}^{n-1}\left(\int_{0}^{1}\left|S_{j}(\alpha)\right|^{\ell_{j}} \mathrm{~d} \alpha\right)^{1 / \ell_{j}} \\
& \ll B^{\varepsilon} \cdot \sup _{\alpha \in \mathfrak{m}}\left|S_{n}(\alpha)\right| \cdot \prod_{j=0}^{n-1}\left(\frac{B}{\gamma_{j}}\right)^{\frac{\ell_{j}-m_{j}}{m_{j} \ell_{j}}},
\end{aligned}
$$

since $H \geq 1$ and $\gamma_{j} \leq B$ for all $0 \leq j \leq n-1$. We apply Lemma 2.5 to estimate $S_{n}(\alpha)$. Finally, observing that

$$
-\frac{1}{m_{j}}+\frac{1}{\ell_{j}} \leq-\frac{1}{m_{j}}+\frac{1}{m_{j}\left(m_{j}+1\right)}=-\frac{1}{m_{j}+1},
$$

for all $0 \leq j \leq n-1$, we obtain

$$
\begin{aligned}
\int_{\mathfrak{m}}\left|\prod_{j=0}^{n} S_{j}(\alpha)\right| \mathrm{d} \alpha & \ll B^{\frac{1}{m_{n}}-\frac{\delta}{m_{n}\left(m_{n}+1\right)}+\varepsilon} \gamma_{n}^{-\frac{1}{m_{n}+1}} \cdot \prod_{j=0}^{n-1} B^{\frac{1}{m_{j}}-\frac{1}{\ell_{j}}} \gamma_{j}^{-\frac{1}{m_{j}+1}} \\
& \ll B^{\Gamma-\frac{\delta}{m_{n}\left(m_{n}+1\right)}+\varepsilon} \prod_{j=0}^{n} \gamma_{j}^{-\frac{1}{m_{j}+1}}
\end{aligned}
$$

as required.

\subsection{Final estimate}

We may now bring together Lemmas 2.2 and 2.6 in (2.4), in order to record the following estimate for the counting function (2.3).

Theorem 2.7 Assume that $2 \leq m_{0} \leq \cdots \leq m_{n}$ and (2.1) holds. Let $\delta$ satisfy (2.5) and let $\varepsilon>0$. Then 


$$
\begin{aligned}
& M_{\mathbf{c} ; \boldsymbol{\gamma}}(B ; \mathbf{h}, H ; N)=\frac{\mathfrak{S}_{\mathbf{c} ; \boldsymbol{\gamma}}(\mathbf{h}, H ; N) \mathfrak{J}_{\mathbf{c}}}{H^{n+1} \prod_{j=0}^{n} \gamma_{j}^{1 / m_{j}}} B^{\Gamma}+O\left(E_{1}(\boldsymbol{\gamma} ; H)+\frac{B^{\Gamma-\delta} E_{2}(\boldsymbol{\gamma} ; H)}{H^{n+1} \prod_{j=0}^{n} \gamma_{j}^{1 / m_{j}}}\right) \\
& +O\left(B^{\Gamma-\frac{\delta}{m_{n}\left(m_{n}+1\right)}}+\varepsilon \prod_{j=0}^{n} \gamma_{j}^{-1 /\left(m_{j}+1\right)}\right),
\end{aligned}
$$

where $E_{1}$ and $E_{2}$ are given by (2.7) and (2.9), respectively.

We end this section by indicating how this implies Theorem 1.4, for which we observe that $M_{\mathbf{c} ; \gamma}(B ; \mathbf{h}, H ; N)=R(N)$ when $H=1, B=N$ and $c_{j}=\gamma_{j}=1$ for $0 \leq j \leq n$. The error term is clearly in the desired shape and recourse to (2.11) shows that $\mathfrak{S}_{\mathbf{c} ; \gamma}(\mathbf{h}, H ; N)=\mathfrak{S}(N)$, with

$$
\mathfrak{S}(N)=\sum_{q=1}^{\infty} \frac{1}{q^{n+1}} \sum_{\substack{0 \leq a<q \\ \operatorname{gcd}(a, q)=1}} e\left(-\frac{a N}{q}\right) \prod_{j=0}^{n} \sum_{0 \leq k<q} e\left(\frac{a}{q} k^{m_{j}}\right) .
$$

Finally, the standard arguments described in Chapter 4 of Davenport [6] readily yield

$$
\mathfrak{J}_{\mathbf{c}}=\int_{-\infty}^{\infty} e(-\lambda) \prod_{j=0}^{n} \int_{0}^{1} e\left(\lambda z^{m_{j}}\right) \mathrm{d} z \mathrm{~d} \lambda=\frac{\prod_{i=0}^{n} \Gamma\left(1+\frac{1}{m_{i}}\right)}{\Gamma\left(\sum_{i=0}^{n} \frac{1}{m_{i}}\right)} .
$$

This therefore completes the proof of Theorem 1.4.

\section{Orbifold Manin: proof of Theorem 1.1}

We now turn to the task of proving an asymptotic formula for the counting function $N\left(\mathbb{P}^{n-1}, \Delta ; B\right)$ in Theorem 1.1. We shall assume without loss of generality that $2 \leq m_{0} \leq$ $\cdots \leq m_{n}$, so that (1.3) implies (2.1). The counting function can be written

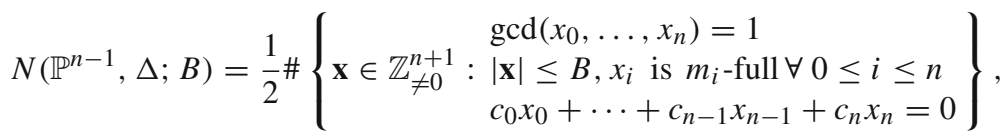

where we henceforth follow the convention that $c_{n}=-1$. In view of (1.7), we may write

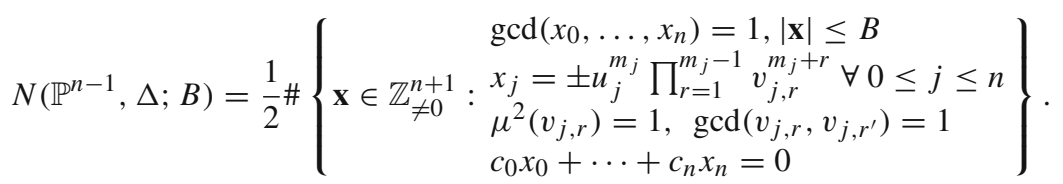

Suppose that we are given vectors $\mathbf{S}$ and $\mathbf{t}$ with coordinates $s_{j} \in \mathbb{N}$ and $t_{j, r} \in \mathbb{N}$ for $0 \leq j \leq n$ and $1 \leq r \leq m_{j}-1$. It will be convenient to introduce the set

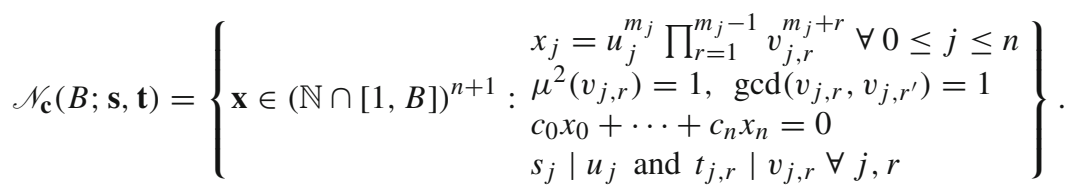


Given $\boldsymbol{\epsilon} \in\{ \pm 1\}^{n+1}$ let $\boldsymbol{\epsilon} \mathbf{c}$ denote the vector with coordinates $\epsilon_{j} c_{j}$. Then

$$
N\left(\mathbb{P}^{n-1}, \Delta ; B\right)=\frac{1}{2} \sum_{\epsilon \in\{ \pm 1\}^{n+1}} \#\left(\mathscr{N}_{\mathbf{\epsilon} \mathbf{c}}(B ; \mathbf{1}, \mathbf{1}) \cap \mathbb{Z}_{\text {prim }}^{n+1}\right),
$$

where 1 is the vector with all coordinates equal to 1 .

We need to develop an inclusion-exclusion argument to cope with the coprimality condition in this expression. To ease notation we replace $\boldsymbol{\epsilon} \mathbf{c}$ by $\mathbf{c}$. Let $\mathbf{x} \in \mathscr{N}_{\mathbf{c}}(B ; \mathbf{1}, \mathbf{1})$. It is clear that $\operatorname{gcd}\left(x_{0}, \ldots, x_{n}\right)>1$ if and only if there exists a prime $p$ and a subset $I \subseteq\{0, \ldots, n\}$ for which $p \mid u_{j}$ for all $j \in I$ and also $p \mid \prod_{r=1}^{m_{j}-1} v_{j, r}$ for all $j \notin I$. (Note that $I$ is allowed to be the empty set here.)

Let $\mathscr{G}$ denote the set of all possible vectors $\mathbf{g} \in \mathbb{N}^{n+1}$ with $1 \leq g_{j} \leq m_{j}-1$ for $0 \leq j \leq n$. Let $\mathrm{P}=\{2,3,5, \ldots\}$ denote the set of primes and let $\mathscr{R}$ be a non-empty finite collection of triples $(\mathbf{g} ; p ; I)$ where $\mathbf{g} \in \mathscr{G}, p \in \mathrm{P}$ and (possibly empty) $I \subseteq\{0, \ldots, n\}$. Let $\mathscr{R}(p)$ be the subset of $\mathscr{R}$ containing all the triples in $\mathscr{R}$ with prime $p$. In what follows we adhere to common convention and stipulate that a union over the empty set is the empty set and a product over the empty set is 1 . We let

$$
I(\mathscr{R}(p))=\bigcup_{(\mathbf{g} ; p ; I) \in \mathscr{R}(p)} I \text { and } J(\mathbf{g} ; \mathscr{R}(p))=\bigcup_{\substack{\left(\mathbf{g}^{\prime} ; p ; I\right) \in \mathscr{R}(p) \\ \mathbf{g}^{\prime}=\mathbf{g}}}\{0, \ldots, n\} \backslash I .
$$

Next, we define $\mathbf{a}(\mathscr{R})$ to be the vector in $\mathbb{N}^{n+1}$ with coordinates

$$
a_{j}=\prod_{\substack{p \in \mathrm{P} \\ j \in I(\mathscr{R}(p))}} p, \quad(0 \leq j \leq n)
$$

and we define $\mathbf{b}(\mathscr{R})$ to be the vector in $\mathbb{N}^{\sum_{j=0}^{n}\left(m_{j}-1\right)}$ with coordinates

$$
b_{j, r}=\prod_{\substack{p \in \mathrm{P} \\ j \in J(\mathbf{g} ; \mathscr{R}(p)) \text { for some } \\ \text { satisfying } g_{j}=r}} p, \quad\left(0 \leq j \leq n, 1 \leq r \leq m_{j}-1\right) .
$$

It is easy to see that $(\mathbf{a}(\mathscr{R}), \mathbf{b}(\mathscr{R})) \neq(\mathbf{1}, \mathbf{1})$ as soon as $\mathscr{R} \neq \varnothing$. Moreover, when $\mathscr{R}=$ $\{(\mathbf{g} ; p ; I)\}$ we see that $\mathscr{N}_{\mathbf{c}}(B ; \mathbf{a}(\mathbf{g} ; p ; I), \mathbf{b}(\mathbf{g} ; p ; I))$ is precisely the set of $\mathbf{x} \in \mathscr{N}_{\mathbf{c}}(B ; \mathbf{1}, \mathbf{1})$ satisfying $p \mid u_{j}$ for all $j \in I$ and $p \mid v_{j, g_{j}}$ for all $j \notin I$. In particular, it is now clear that

$$
\mathscr{N}_{\mathbf{c}}(B ; \mathbf{1}, \mathbf{1}) \cap \mathbb{Z}_{\mathrm{prim}}^{n+1}=\mathscr{N}_{\mathbf{c}}(B ; \mathbf{1}, \mathbf{1}) \backslash \bigcup_{\substack{\mathbf{g} \in \mathscr{G} \\ p \in \mathrm{P} \\ I \subseteq\{0, \ldots, n\}}} \mathscr{N}_{\mathbf{c}}(B ; \mathbf{a}(\mathbf{g} ; p ; I), \mathbf{b}(\mathbf{g} ; p ; I)) .
$$

We proceed by establishing the following result.

Lemma 3.1 Given any $\mathscr{R} \neq \emptyset$, we have

$$
\mathscr{N}_{\mathbf{c}}(B ; \mathbf{a}(\mathscr{R}), \mathbf{b}(\mathscr{R}))=\bigcap_{(\mathbf{g} ; p ; I) \in \mathscr{R}} \mathscr{N}_{\mathbf{c}}(B ; \mathbf{a}(\mathbf{g} ; p ; I), \mathbf{b}(\mathbf{g} ; p ; I)) .
$$

Proof Let $\mathbf{x}$ belong to the intersection on the right hand side. Then, given any $(\mathbf{g} ; p ; I) \in \mathscr{R}$, we have $p \mid u_{j}$ for all $j \in I$ and $p \mid v_{j, r}$ if $j \notin I$ and $r=g_{j}$, where $x_{j}=u_{j}^{m_{j}} \prod_{r=1}^{m_{j}-1} v_{j, r}^{m_{j}+r}$. 
Therefore, $p \mid u_{j}$ for all $p$ such that $j \in I(\mathscr{R}(p))$ and $p \mid v_{j, r}$ for all $p$ such that

$$
j \in \bigcup_{\substack{(\mathbf{g} ; p ; I) \in \mathscr{R}(p) \\ g_{j}=r}}\{0, \ldots, n\} \backslash I .
$$

Thus (3.2) and (3.3) imply that $a_{j} \mid u_{j}$ and $b_{j, r} \mid v_{j, r}$, whence it follows that $\mathbf{x} \in \mathscr{N}_{\mathbf{c}}(B ; \mathbf{a}(\mathscr{R}), \mathbf{b}(\mathscr{R}))$. On the other hand, if $\mathbf{x} \in \mathscr{N}_{\mathbf{c}}(B ; \mathbf{a}(\mathscr{R}), \mathbf{b}(\mathscr{R}))$ then we may reverse the argument to deduce that $\mathbf{x}$ also belongs to the intersection of all the sets $\mathscr{N}_{\mathbf{c}}(B ; \mathbf{a}(\mathbf{g} ; p ; I), \mathbf{b}(\mathbf{g} ; p ; I))$ for $(\mathbf{g} ; p ; I) \in \mathscr{R}$. This completes the proof of the lemma.

Given vectors $\mathbf{s}$ and $\mathbf{t}$ composed from positive integers, let

$$
\varpi(\mathbf{s}, \mathbf{t})=\sum_{k=1}^{\infty}(-1)^{k} \#\{\mathscr{R}: \# \mathscr{R}=k,(\mathbf{s}, \mathbf{t})=(\mathbf{a}(\mathscr{R}), \mathbf{b}(\mathscr{R}))\} .
$$

We henceforth set

$$
\varpi(\mathbf{1}, \mathbf{1})=1 .
$$

Then, on combining the inclusion-exclusion principle with Lemma 3.1, we obtain

$$
\begin{aligned}
\# \bigcup_{\substack{\mathbf{g} \in \mathscr{G} \\
p \in \mathbb{P} \\
I \subseteq\{0, \ldots, n\}}} \mathscr{N}_{\mathbf{c}}(B ; \mathbf{a}(\mathbf{g} ; p ; I), \mathbf{b}(\mathbf{g} ; p ; I)) & =-\sum_{k=1}^{\infty}(-1)^{k} \sum_{\# \mathscr{R}=k} \# \mathscr{N}_{\mathbf{c}}(B ; \mathbf{a}(\mathscr{R}), \mathbf{b}(\mathscr{R})) \\
& =-\sum_{(\mathbf{s}, \mathbf{t}) \neq(\mathbf{1}, \mathbf{1})} \varpi(\mathbf{s}, \mathbf{t}) \cdot \# \mathscr{N}_{\mathbf{c}}(B ; \mathbf{s}, \mathbf{t}) .
\end{aligned}
$$

Note that $\# \mathscr{N}_{\mathbf{c}}(B ; \mathbf{a}(\mathscr{R}), \mathbf{b}(\mathscr{R}))=0$ when $\# \mathscr{R}$ is sufficiently large with respect to $B$. Bringing this together with (3.4), we conclude that

$$
\# \mathscr{N}_{\mathbf{c}}(B ; \mathbf{1}, \mathbf{1}) \cap \mathbb{Z}_{\mathrm{prim}}^{n+1}=\sum_{\mathbf{s}, \mathbf{t}} \varpi(\mathbf{s}, \mathbf{t}) \cdot \# \mathscr{N}_{\mathbf{c}}(B ; \mathbf{s}, \mathbf{t}) .
$$

It remains to asymptotically estimate these quantities.

We collect together some properties of the function $\varpi(\mathbf{s}, \mathbf{t})$.

Lemma 3.2 Let $(\mathbf{s}, \mathbf{t}) \neq(\mathbf{1}, \mathbf{1})$ and let $p \in P$. We let $\mathbf{s}^{[p]}$ be the vector whose $j$ th coordinate is $s_{j}^{[p]}=p^{\mathrm{val}_{p}\left(s_{j}\right)}$ and $\mathbf{t}^{[p]}$ be the vector whose $(j, r)$ th coordinate is $t_{j, r}^{[p]}=p^{\operatorname{val}_{p}\left(t_{j, r}\right)}$. Then the following are true:

(i) $\varpi(\mathbf{s}, \mathbf{t})=\prod_{p \in P} \varpi\left(\mathbf{s}^{[p]}, \mathbf{t}^{[p]}\right)$;

(ii) $\varpi(\mathbf{s}, \mathbf{t})=0$ if one of the coordinates of $\mathbf{s}$ or $\mathbf{t}$ is divisible by $p^{2}$;

(iii) $\varpi(\mathbf{s}, \mathbf{t})=0$ if one of the coordinates of $\mathbf{s}$ or $\mathbf{t}$ is divisible by $p$, but there exists $0 \leq j \leq n$ with $s_{j}^{[p]}=t_{j, 1}^{[p]}=\cdots=t_{j, m_{j}-1}^{[p]}=1$; and

(iv) $\varpi\left(\mathbf{s}^{[p]}, \mathbf{t}^{[p]}\right) \ll 1$.

Proof It follows from the definitions of $\mathbf{a}(\mathscr{R})$ and $\mathbf{b}(\mathscr{R})$ that

$$
\mathbf{a}(\mathscr{R})=\prod_{p \in \mathrm{P}} \mathbf{a}(\mathscr{R}(p)) \quad \text { and } \quad \mathbf{b}(\mathscr{R})=\prod_{p \in \mathrm{P}} \mathbf{b}(\mathscr{R}(p)),
$$


where we define multiplication of vectors by multiplying the corresponding coordinates. We clearly have $(\mathbf{s}, \mathbf{t})=\prod_{p \in \mathrm{P}}\left(\mathbf{s}^{[p]}, \mathbf{t}^{[p]}\right)$ and $\# \mathscr{R}=\sum_{p \in \mathrm{P}} \# \mathscr{R}(p)$. Thus

$$
\prod_{\substack{p \in \mathrm{P} \\\left(\mathbf{s}^{[p]}, \mathbf{t}^{[p]}\right) \neq(\mathbf{1}, \mathbf{1})}} \varpi\left(\mathbf{s}^{[p]}, \mathbf{t}^{[p]}\right)=\prod_{p \in \mathrm{P}} \sum_{k^{\prime}=1}^{\infty}(-1)^{k^{\prime}} T_{p}\left(k^{\prime}\right),
$$

where

$$
T_{p}\left(k^{\prime}\right)=\#\left\{\mathscr{R} \subseteq \mathscr{G} \times\{p\} \times\{0, \ldots, n\}: \begin{array}{l}
\# \mathscr{R}=k^{\prime} \\
\left(\mathbf{s}^{[p]}, \mathbf{t}^{[p]}\right)=(\mathbf{a}(\mathscr{R}), \mathbf{b}(\mathscr{R}))
\end{array}\right\} .
$$

It follows that

$$
\prod_{\substack{p \in \mathrm{P} \\\left(\mathbf{s}^{[p]}, \mathbf{t}^{[p]}\right) \neq(\mathbf{1}, \mathbf{1})}} \varpi\left(\mathbf{s}^{[p]}, \mathbf{t}^{[p]}\right)=\sum_{k=1}^{\infty}(-1)^{k} \sum_{\sum k_{p}=k} \prod_{\substack{p \in \mathrm{P} \\ k_{p}>0}} T_{p}\left(k_{p}\right)=\varpi(\mathbf{s}, \mathbf{t}),
$$

which thereby establishes (i).

To prove (ii) we note that it is not possible for $p^{2}$ to divide any coordinate of $\mathbf{a}(\mathscr{R}(p))$ or $\mathbf{b}(\mathscr{R}(p))$ for any prime $p$ and $\mathscr{R} \neq \emptyset$. Thus $\varpi\left(\mathbf{s}^{[p]}, \mathbf{t}^{[p]}\right)=0$ if one of the coordinates of $\mathbf{s}^{[p]}$ or $\mathbf{t}^{[p]}$ is divisible by $p^{2}$.

Next, to prove (iii) let $(\mathbf{s}, \mathbf{t}) \neq(\mathbf{1}, \mathbf{1})$ and assume without loss of generality that $p \mid$ $s_{1} t_{1,1} \ldots t_{1, m_{1}-1}$ and $s_{2}^{[p]}=t_{2,1}^{[p]}=\cdots=t_{2, m_{2}-1}^{[p]}=1$. Suppose there exists $\mathscr{R}$ such that $(\mathbf{s}, \mathbf{t})=(\mathbf{a}(\mathscr{R}), \mathbf{b}(\mathscr{R}))$. Then we have $\mathscr{R}(p) \not \varnothing$, and also

$$
2 \in\{0, \ldots, n\}=I(\mathscr{R}(p)) \cup \bigcup_{\mathbf{g} \in \mathscr{G}} J(\mathbf{g} ; \mathscr{R}(p)) .
$$

If $2 \in I(\mathscr{R}(p))$ then $p \mid s_{2}$. On the other hand, if $2 \in J(\mathbf{g} ; \mathscr{R}(p))$ then $p \mid t_{2, g_{2}}$. In either case we have a contradiction, whence $\varpi(\mathbf{s}, \mathbf{t})=0$.

Finally, to prove (iv) we note there are only $O(1)$ options for $\mathscr{R}(p)$ for any fixed $p \in \mathrm{P}$. It now follows from the definition that

$$
\left|\varpi\left(\mathbf{s}^{[p]}, \mathbf{t}^{[p]}\right)\right| \leq \sum_{k=1}^{\infty} \#\{\mathscr{R}(p): \# \mathscr{R}(p)=k\} \ll 1,
$$

as required.

Given $\varepsilon>0$, it follows from Lemma 3.2 that

$$
\varpi(\mathbf{s}, \mathbf{t}) \ll \prod_{j=0}^{n} s_{j}^{\varepsilon} \prod_{1 \leq r \leq m_{j}-1} t_{j, r}^{\varepsilon} .
$$

We now proceed by studying

$$
\sum_{\mathbf{s}, \mathbf{t}} \varpi(\mathbf{s}, \mathbf{t}) \cdot \# \mathscr{N}_{\mathbf{c}}(B ; \mathbf{s}, \mathbf{t})
$$

Let

$$
\gamma_{j}=s_{j}^{m_{j}} \prod_{r=1}^{m_{j}-1} t_{j, r}^{m_{j}+r} v_{j, r}^{m_{j}+r}
$$


Then

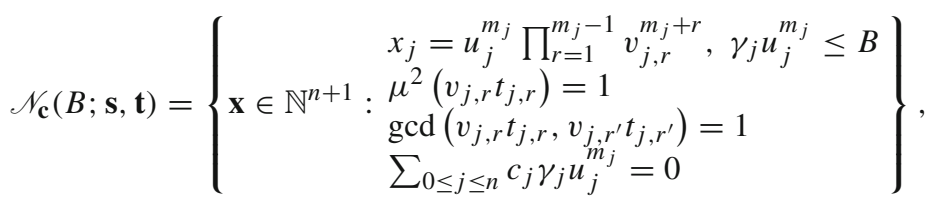

where the indices run over $0 \leq j \leq n$ and $1 \leq r<r^{\prime} \leq m_{j}-1$. For each $\mathbf{s}$ and $\mathbf{t}$ we let

$$
\sum_{\mathbf{v}}^{(1)}
$$

denote the sum over all $\mathbf{v}$ satisfying $\gamma_{j} \leq B, \operatorname{gcd}\left(v_{j, r} t_{j, r}, v_{j, r^{\prime}} t_{j, r^{\prime}}\right)=1$ and $\mu^{2}\left(v_{j, r} t_{j, r}\right)=$ 1. (If there is no $\mathbf{v}$ which satisfies the above conditions then the sum is considered to be 0 .) We may now write

$$
\# \mathscr{N}_{\mathbf{c}}(B ; \mathbf{s}, \mathbf{t})=\sum_{\mathbf{v}}^{(1)} M_{\mathbf{c} ; \boldsymbol{\gamma}}(B),
$$

where $M_{\mathbf{c} ; \gamma}(B)=M_{\mathbf{c} ; \gamma}(B ; \mathbf{0}, 1 ; 0)$, in the notation (2.3). Guided by Lemma 3.2, we let

$$
\sum_{\mathbf{s}, \mathbf{t}}^{(2)}
$$

denote the sum over $\mathbf{s}, \mathbf{t}$ satisfying $s_{j}^{m_{j}} \prod_{r=1}^{m_{j}-1} t_{j, r}^{m_{j}+r} \leq B$ and $\operatorname{gcd}\left(t_{j, r}, t_{j, r^{\prime}}\right)=1$, together with the condition that none of the coordinates of $\mathbf{s}$ or $\mathbf{t}$ is divisible by $p^{2}$ for any prime $p$ and if one of the coordinates of $\mathbf{s}$ or $\mathbf{t}$ is divisible by $p$ then $p \mid s_{j} t_{j, 1} \ldots t_{j, m_{j}-1}$ for all $0 \leq j \leq n$.

We want to apply Theorem 2.7 with $H=1$ and $N=0$. Let $\delta>0$ satisfy (2.5) and let $\mathfrak{S}_{\mathbf{c} ; \gamma}=\mathfrak{S}_{\mathbf{c} ; \boldsymbol{\gamma}}(\mathbf{0}, 1 ; 0)$. Then, on appealing to Lemma 3.2 and (3.6), we deduce that

$$
\sum_{\mathbf{s}, \mathbf{t}} \varpi(\mathbf{s}, \mathbf{t}) \cdot \# \mathscr{N}_{\mathbf{c}}(B ; \mathbf{s}, \mathbf{t})=M(B)+O\left(B^{\Gamma+\varepsilon} \sum_{i=1}^{3} F_{i}(B)\right),
$$

for any $\varepsilon>0$, where

$$
M(B)=B^{\Gamma} \sum_{\mathbf{s}, \mathbf{t}}^{(2)} \varpi(\mathbf{s}, \mathbf{t}) \sum_{\mathbf{v}}^{(1)} \mathfrak{S}_{\mathbf{c} ; \gamma} \mathfrak{J}_{\mathbf{c}} \prod_{j=0}^{n} \gamma_{j}^{-1 / m_{j}} .
$$

Moreover, in view of (2.7) and (2.9), the error terms are given by

$$
\begin{aligned}
& F_{1}(B)=B^{(2 n+5) \delta} \sum_{k=0}^{n} B^{-1 / m_{k}} \sum_{\mathbf{s}, \mathbf{t}}^{(2)} \sum_{\mathbf{v}}^{(1)} \prod_{\substack{j=0 \\
j \neq k}}^{n} \gamma_{j}^{-1 / m_{j}}, \\
& F_{2}(B)=B^{-\delta} \sum_{\mathbf{s}, \mathbf{t}}^{(2)} \sum_{\mathbf{v}}^{(1)} \sum_{q=1}^{\infty} q^{1-\Gamma+\varepsilon} \prod_{j=0}^{n} \frac{\operatorname{gcd}\left(\gamma_{j}, q\right)^{1 / m_{j}}}{\gamma_{j}^{1 / m_{j}}}, \\
& F_{3}(B)=B^{-\frac{\delta}{m_{n}\left(m_{n}+1\right)}} \sum_{\mathbf{s}, \mathbf{t}}^{(2)} \sum_{\mathbf{v}}^{(1)} \prod_{j=0}^{n} \gamma_{j}^{-1 /\left(m_{j}+1\right)} .
\end{aligned}
$$


We now need to estimate these three error terms. In doing so it will be convenient to set $w_{j}=v_{j, 1}^{m_{j}+1} \ldots v_{j, m_{j}-1}^{2 m_{j}-1}$ and $\tau_{j}=s_{j}^{m_{j}} \prod_{r=1}^{m_{j}-1} t_{j, r}^{m_{j}+r}$.

Now for any $\tau \geq 1$, we have

$$
\sum_{v_{1}^{m+1} \ldots v_{m-1}^{2 m-1} \leq B / \tau} 1 \ll \sum_{v_{2}, \ldots, v_{m-1}=1}^{\infty}\left(\frac{B / \tau}{v_{2}^{m+2} \ldots v_{m-1}^{2 m-1}}\right)^{1 /(m+1)} \ll\left(\frac{B}{\tau}\right)^{1 /(m+1)} .
$$

Similarly,

$$
\sum_{v_{1}^{m+1} \ldots v_{m-1}^{2 m-1} \leq B / \tau}\left(\frac{1}{v_{1}^{m+1} \ldots v_{m-1}^{2 m-1}}\right)^{1 / m} \ll 1 .
$$

Using these estimates it follows that

$$
\begin{aligned}
F_{1}(B) & \ll B^{(2 n+5) \delta} \sum_{k=0}^{n} B^{-1 / m_{k}} \sum_{\mathbf{s}, \mathbf{t}}^{(2)}\left(\frac{B}{\tau_{k}}\right)^{1 /\left(m_{k}+1\right)} \prod_{\substack{j=0 \\
j \neq k}}^{n} \tau_{j}^{-1 / m_{j}} \\
& \ll B^{-1 / m_{n}\left(m_{n}+1\right)+(2 n+5) \delta} \sum_{\mathbf{s}, \mathbf{t}}^{(2)} \prod_{j=0}^{n} \tau_{j}^{-1 /\left(m_{j}+1\right)},
\end{aligned}
$$

where we recall that $\tau_{j}=s_{j}^{m_{j}} \prod_{r=1}^{m_{j}-1} t_{j, r}^{m_{j}+r}$. Lemma 3.2 now yields

$$
\sum_{\mathbf{s}, \mathbf{t}}^{(2)} \prod_{j=0}^{n} \tau_{j}^{-1 /\left(m_{j}+1\right)} \leq \prod_{p}\left(1+\prod_{j=0}^{n}\left(2 m_{j}-1\right) p^{-m_{j} /\left(m_{j}+1\right)}\right) \ll 1,
$$

since $\sum_{j=0}^{n} m_{j} /\left(m_{j}+1\right)>1$. (Note that the factor $2 m_{j}-1$ on the right hand side comes from taking into account the $2 m_{j}-1$ possibilities where the factor $p$ appears in $s_{j}$ or $t_{j}$.) We have therefore shown that

$$
F_{1}(B) \ll B^{-1 / m_{n}\left(m_{n}+1\right)+(2 n+5) \delta} .
$$

Turning to the estimation of $F_{2}(B)$, we may write

$$
F_{2}(B) \leq B^{-\delta} \sum_{q=1}^{\infty} q^{1-\Gamma+\varepsilon} f_{1}(q) f_{2}(q)
$$

where

$$
\begin{aligned}
& f_{1}(q)=\sum_{\mathbf{s}, \mathbf{t}}^{(2)} \prod_{j=0}^{n}\left(\frac{\operatorname{gcd}\left(s_{j}^{m_{j}} t_{j, 1}^{m_{j}+1} \ldots t_{j, m_{j}-1}^{2 m_{j}-1}, q\right)}{s_{j}^{m_{j}} t_{j, 1}^{m_{j}+1} \ldots t_{j, m_{j}-1}^{2 m_{j}-1}}\right)^{1 / m_{j}}, \\
& f_{2}(q)=\sum_{\substack{w_{j} \leq B \\
\mu^{2}\left(v_{j, r}\right)=1}} \prod_{j=0}^{n} \frac{\operatorname{gcd}\left(w_{j}, q\right)^{1 / m_{j}}}{w_{j}^{1 / m_{j}}} .
\end{aligned}
$$

We first show that

$$
\sum_{x \leq B^{1 /(m+r)}} \frac{\mu^{2}(x) \operatorname{gcd}\left(x^{m+r}, q\right)^{1 / m}}{x^{(m+r) / m}} \ll q^{\varepsilon},
$$


if $r \geq 1$. To see this we note that the left hand side is at most

$$
\sum_{d \mid q} d^{1 / m} \sum_{\substack{x \leq B^{1 /(m+r)} \\ d \mid x^{m+r}}} \frac{\mu^{2}(x)}{x^{(m+r) / m}}
$$

When $\mu^{2}(x)=1$, any $d \mid x^{m+r}$ admits a factorisation $d=d_{1} d_{2}^{2} \ldots d_{m+r}^{m+r}$ such that $d_{1} \ldots d_{m+r} \mid x$, where $\mu^{2}\left(d_{i}\right)=1$ and $\operatorname{gcd}\left(d_{i}, d_{j}\right)=1$ for $i \neq j$. If we write $x=x^{\prime} d_{1} \ldots d_{m+r}$, then this sum is

$$
\leq \sum_{\substack{d \mid q \\ d=d_{1} \ldots d_{m+r}^{m+r}}} \frac{\left(d_{1} \ldots d_{m+r}^{m+r}\right)^{1 / m}}{\left(d_{1} \ldots d_{m+r}\right)^{(m+r) / m}} \sum_{x^{\prime} \leq B^{1 /(m+r) /\left(d_{1} \ldots d_{m+r}\right)}} \frac{1}{x^{\prime(m+r) / m}} .
$$

The inner $x^{\prime}$-sum is absolutely convergent since $r \geq 1$. The remaining sum over $d \mid q$ is $O\left(q^{\varepsilon}\right)$ for any $\varepsilon>0$, by the standard estimate for the divisor function. This therefore establishes (3.9).

An application of (3.9) immediately yields

$$
f_{2}(q) \leq \prod_{j=0}^{n} \prod_{r=1}^{m_{j}-1} \sum_{v_{j, r} \leq B^{1 /\left(m_{j}+r\right)}} \frac{\mu^{2}\left(v_{j, r}\right) \operatorname{gcd}\left(v_{j, r}^{m_{j}+r}, q\right)^{1 / m_{j}}}{v_{j, r}^{\left(m_{j}+r\right) / m_{j}}} \ll q^{\varepsilon},
$$

for any $\varepsilon>0$. Next, let

$$
f_{1, T}(q)=\sum_{\substack{\mathbf{s}, \mathbf{t} \\ \max \left\{\tau_{0}, \ldots, \tau_{n}\right\} \geq T}}^{(2)} \prod_{j=0}^{n} \tau_{j}^{\varepsilon}\left(\frac{\operatorname{gcd}\left(\tau_{j}, q\right)}{\tau_{j}}\right)^{1 / m_{j}}
$$

for any $\varepsilon>0$ and $T \geq 1$, where $\tau_{j}=s_{j}^{m_{j}} \prod_{r=1}^{m_{j}-1} t_{j, r}^{m_{j}+r}$. In particular we have $f_{1}(q) \leq$ $f_{1,1}(q)$. We claim that

$$
f_{1, T}(q) \ll q^{6 \varepsilon\left(m_{0}+\cdots+m_{n}\right)} T^{-\varepsilon}
$$

for any sufficiently small $\varepsilon>0$. Once achieved, it will follow that

$$
F_{2}(B) \ll B^{-\delta},
$$

since (2.10) implies that $\Gamma-1>1$.

To check the claim we let $\mathscr{T}$ denote the set of vectors $\left(\tau_{0}, \ldots, \tau_{n}\right) \in \mathbb{N}^{n+1}$ with the property that for any prime $p$ we have $\operatorname{val}_{p}\left(\tau_{j}\right) \in\left\{0, m_{j}, \ldots, 3 m_{j}-1\right\}$ and, furthermore, if $p \mid \tau_{0} \ldots \tau_{n}$ then $\operatorname{val}_{p}\left(\tau_{j}\right)>0$ for all $0 \leq j \leq n$. Associated to any $\left(\tau_{0}, \ldots, \tau_{n}\right) \in \mathscr{T}$ is a unique choice for $\mathbf{s}, \mathbf{t}$. Thus we find that

$$
\begin{aligned}
f_{1, T}(q) & \ll \frac{1}{T^{\varepsilon}} \sum_{\left(\tau_{0}, \ldots, \tau_{n}\right) \in \mathscr{T}} \prod_{j=0}^{n} \tau_{j}^{2 \varepsilon}\left(\frac{\operatorname{gcd}\left(\tau_{j}, q\right)}{\tau_{j}}\right)^{1 / m_{j}} \\
& \ll \frac{1}{T^{\varepsilon}} \prod_{p}\left(1+\prod_{j=0}^{n} \sum_{m_{j} \leq \alpha_{j} \leq 3 m_{j}-1} p^{\min \left(\alpha_{j}, \operatorname{val}_{p}(q)\right) / m_{j}-\alpha_{j} / m_{j}+2 \varepsilon \alpha_{j}}\right) .
\end{aligned}
$$

When $p \nmid q$ the corresponding local factor takes the shape

$$
1+O\left(p^{-n-1+2 \varepsilon\left(m_{0}+\cdots+m_{n}\right)}\right) \text {. }
$$


Alternatively, when $p \mid q$ the factor is $O\left(p^{6 \varepsilon\left(m_{0}+\cdots+m_{n}\right)}\right)$ Assuming that $\varepsilon$ is sufficiently small this therefore concludes the proof of (3.11).

Finally we must analyse

$$
F_{3}(B)=B^{-\frac{\delta}{m_{n}\left(m_{n}+1\right)}} \sum_{\mathbf{s}, \mathbf{t}}^{(2)} \sum_{\mathbf{v}}^{(1)} \prod_{j=0}^{n} \gamma_{j}^{-1 /\left(m_{j}+1\right)} .
$$

We note that

$$
\sum_{v_{1}^{m+1} \ldots v_{m-1}^{2 m-1} \leq B}\left(\frac{1}{v_{1}^{m+1} \ldots v_{m-1}^{2 m-1}}\right)^{1 /(m+1)} \ll \log B .
$$

Applying (3.8) to handle the resulting sum over $\mathbf{s}$ and $\mathbf{t}$ it easily follows that

$$
F_{3}(B) \ll B^{-\frac{\delta}{m_{n}\left(m_{n}+1\right)}+\varepsilon},
$$

for any $\varepsilon>0$.

We substitute our bounds for the error terms back into (3.7). This yields

$$
\begin{aligned}
& \sum_{\mathbf{s}, \mathbf{t}} \varpi(\mathbf{s}, \mathbf{t}) \cdot \# \mathscr{N}_{\mathbf{c}}(B ; \mathbf{s}, \mathbf{t}) \\
& \quad=M(B)+O\left(B^{\Gamma+\varepsilon}\left\{B^{-\frac{1}{m_{n}\left(m_{n}+1\right)}+(2 n+5) \delta}+B^{-\frac{\delta}{m_{n}\left(m_{n}+1\right)}}\right\}\right) .
\end{aligned}
$$

Remark 3.3 Let us rerun the above argument, with the special choice $(\mathbf{s}, \mathbf{t})=(\mathbf{1}, \mathbf{1})$, since it will be used again in the proof of Theorem 1.3. The starting point is to write

$$
\# \mathscr{N}_{\mathbf{c}}(B ; \mathbf{1}, \mathbf{1})=\sum_{\mathbf{v}}^{(1)} M_{\mathbf{c} ; \gamma}(B),
$$

where now $\boldsymbol{\gamma}$ has components $\gamma_{j}=\prod_{r=1}^{m_{j}-1} v_{j, r}^{m_{j}+r}$. Tracing through the argument, this ultimately leads to the conclusion

$$
\# \mathscr{N}_{\mathbf{c}}(B ; \mathbf{1}, \mathbf{1})=\tilde{M}(B)+O\left(B^{\Gamma+\varepsilon}\left\{B^{-\frac{1}{m_{n}\left(m_{n}+1\right)}+(2 n+5) \delta}+B^{-\frac{\delta}{m_{n}\left(m_{n}+1\right)}}\right\}\right),
$$

for any $\varepsilon>0$, where

$$
\tilde{M}(B)=B^{\Gamma} \sum_{\mathbf{v}}^{(1)} \mathfrak{S}_{\mathbf{c} ; \gamma} \mathfrak{J}_{\mathbf{c}} \prod_{j=0}^{n} \gamma_{j}^{-1 / m_{j}} .
$$

We are now ready to complete the proof of Theorem 1.1. Repeating the arguments used in (3.11) during our analysis of $F_{2}(B)$, it is easy to remove the constraint $s_{j}^{m_{j}} \prod_{r=1}^{m_{j}-1} t_{j, r}^{m_{j}+r} \leq B$ from the summation over $\mathbf{s}, \mathbf{t}$ in the main term $M(B)$. The total error in doing this is $O\left(B^{\Gamma-\eta_{1}}\right)$, for some $\eta_{1}>0$. We proceed under the assumption that (2.1) holds and $\delta$ satisfies (2.5). We may combine (3.1) and (3.5) with (3.12) in order to conclude that $N\left(\mathbb{P}^{n-1}, \Delta ; B\right)$ is

$$
c_{B} B^{\Gamma}+O\left(B^{\Gamma+\varepsilon}\left\{B^{-\frac{1}{m_{n}\left(m_{n}+1\right)}+(2 n+5) \delta}+B^{-\frac{\delta}{m_{n}\left(m_{n}+1\right)}}+B^{-\eta_{1}}\right\}\right),
$$

for any $\varepsilon>0$, where

$$
c_{B}=\frac{1}{2} \sum_{\boldsymbol{\epsilon} \in\{ \pm 1\}^{n+1}} \mathfrak{J}_{\boldsymbol{\epsilon} \mathbf{c}} \sum_{\mathbf{s}, \mathbf{t}} \varpi(\mathbf{s}, \mathbf{t}) \sum_{\mathbf{v}}^{(1)} \frac{\mathfrak{S}_{\boldsymbol{\epsilon} \mathbf{c} ; \boldsymbol{\gamma}}}{\prod_{j=0}^{n} \gamma_{j}^{1 / m_{j}}} .
$$


The error term is of the shape claimed in Theorem 1.1 and so it remains to analyse the quantity $c_{B}$.

The dependence on $B$ in the factor $c_{B}$ arises from the definition of the sum $\sum^{(1)}$. A straightforward repetition of our arguments above suffice to show that

$$
c_{B}=c+O\left(B^{-\eta_{2}}\right)
$$

for some $\eta_{2}>0$, where $c$ is the constant that is defined as in $c_{B}$, but with the summation conditions $\gamma_{j} \leq B$ removed from $\sum^{(1)}$, for $0 \leq j \leq n$. This shows that $N\left(\mathbb{P}^{n-1}, \Delta ; B\right)=$ $c B^{\Gamma}+O\left(B^{\Gamma-\eta}\right)$ for an appropriate $\eta>0$, as claimed in Theorem 1.1. To go further, we adopt the notation $\mathbf{s}^{\mathbf{m}} \mathbf{w}=\left(s_{0}^{m_{0}} w_{0}, \ldots, s_{n}^{m_{n}} w_{n}\right)$, where we recall that $w_{j}=v_{j, 1}^{m_{j}+1} \ldots v_{j, m_{j}-1}^{2 m_{j}-1}$ for $0 \leq j \leq n$. Changing the order of summation, we may write

$$
c=\frac{1}{2} \sum_{\boldsymbol{\epsilon} \in\{ \pm 1\}^{n+1}} \mathfrak{J}_{\mathbf{v} \in \mathbf{c}} \sum_{\mathbf{v} \in \mathbb{N}_{j=0}^{n}} \frac{\prod_{j=0}^{n} \mu^{2}\left(v_{j, 1} \ldots v_{\left.j, m_{j}-1\right)}\right)}{\prod_{j=0}^{n} w_{j}^{1 / m_{j}}} \sum_{\substack{\mathbf{s}, \mathbf{t} \\ \mathbf{t} \mid \mathbf{v}}} \varpi(\mathbf{s}, \mathbf{t}) \frac{\mathfrak{S}_{\boldsymbol{\epsilon} \mathbf{c} ; \mathbf{s}^{\mathbf{m}} \mathbf{w}}}{\prod_{j=0}^{n} s_{j}},
$$

with the understanding that $\mathbf{t} \mid \mathbf{v}$ means $t_{j, r} \mid v_{j, r}$ for all $j$ and $r$. We claim that

$$
\sum_{\substack{\mathbf{s}, \mathbf{t} \\ \mathbf{t} \mid \mathbf{v}}} \varpi(\mathbf{s}, \mathbf{t}) \frac{\mathfrak{S}_{\boldsymbol{\epsilon} \mathbf{c} ; \mathbf{s}^{\mathbf{m}} \mathbf{w}}}{\prod_{j=0}^{n} s_{j}}=\prod_{p}\left(\lim _{T \rightarrow \infty} \frac{\mathscr{M}_{\boldsymbol{\epsilon}, T}(\mathbf{v}, p)}{p^{n T}}\right),
$$

where

$$
\mathscr{M}_{\boldsymbol{\epsilon}, T}(\mathbf{v}, p)=\#\left\{\mathbf{k} \bmod p^{T}: \begin{array}{l}
\sum_{j=0}^{n} \epsilon_{j} c_{j} w_{j} k_{j}^{m_{j}} \equiv 0 \bmod p^{T} \\
\exists j \text { such that } p \nmid k_{j} v_{j, 1} \ldots v_{j, m_{j}-1}
\end{array}\right\} .
$$

This will complete our analysis of the leading constant $c$ in Theorem 1.1.

To check the claim we put $c_{j}^{\prime}=\epsilon_{j} c_{j}$ for $0 \leq j \leq n$. It follows from (2.11) and multiplicativity that

$$
\frac{\mathfrak{S}_{\mathbf{c}^{\prime} ; \mathbf{s}^{\mathbf{m}_{\mathbf{w}}}}}{\prod_{j=0}^{n} s_{j}}=\prod_{p} \frac{1}{\prod_{j=0}^{n} s_{j}^{[p]}}\left(1+\sum_{t=1}^{\infty} \mathscr{B}_{\mathbf{s}^{\mathbf{m}} \mathbf{w}}\left(p^{t}\right)\right),
$$

where

$$
\mathscr{B}_{\mathbf{s}^{\mathbf{m}} \mathbf{w}}\left(p^{t}\right)=\frac{1}{p^{t(n+1)}} \sum_{\substack{0 \leq a<p^{t} \\ \operatorname{gcd}\left(a, p^{t}\right)=1}} \prod_{j=0}^{n} \sum_{0 \leq k<p^{t}} e\left(\frac{a}{p^{t}} c_{j}^{\prime} s_{j}^{m_{j}} w_{j} k^{m_{j}}\right) .
$$

Letting

$$
N\left(p^{T}\right)=\#\left\{\mathbf{k} \bmod p^{T}: \sum_{j=0}^{n} c_{j}^{\prime} s_{j}^{m_{j}} w_{j} k_{j}^{m_{j}} \equiv 0 \bmod p^{T}\right\}
$$

we deduce that

$$
\frac{\mathfrak{S}_{\mathbf{c}^{\prime} ; \mathbf{s}^{\mathbf{m}} \mathbf{w}}}{\prod_{j=0}^{n} s_{j}}=\prod_{\substack{p \\ p \nmid s_{0} \ldots s_{n}}}\left(\lim _{T \rightarrow \infty} \frac{N\left(p^{T}\right)}{p^{n T}}\right) \prod_{\substack{p \\ p \mid s_{0} \ldots s_{n}}}\left(\lim _{T \rightarrow \infty} \frac{N\left(p^{T}\right)}{p^{n T} \prod_{j=0}^{n} s_{j}^{[p]}}\right) .
$$


Next, we put

$$
\mathscr{X}_{p, T}(\mathbf{s}, \mathbf{t})=\left\{\mathbf{k} \bmod p^{T}: \begin{array}{l}
\sum_{j=0}^{n} c_{j}^{\prime} w_{j} k_{j}^{m_{j}} \equiv 0 \bmod p^{T} \\
p\left|s_{j} \Rightarrow p\right| k_{j}
\end{array}\right\},
$$

for any $(\mathbf{s}, \mathbf{t})$ such that $(\mathbf{s}, \mathbf{t})=\left(\mathbf{s}^{[p]}, \mathbf{t}^{[p]}\right)$. It is clear that $N\left(p^{T}\right)=\# \mathscr{X}_{p, T}(\mathbf{s}, \mathbf{t})$ when $p \nmid s_{0} \ldots s_{n}$ and that $N\left(p^{t}\right) / \prod_{j=0}^{n} s_{j}^{[p]}=\# \mathscr{X}_{p, T}(\mathbf{s}, \mathbf{t})$ when $p \nmid s_{0} \ldots s_{n}$. It follows that

$$
\frac{\mathfrak{S}_{\mathbf{c}^{\prime} ; \mathbf{s}^{\mathbf{m}} \mathbf{w}}}{\prod_{j=0}^{n} s_{j}}=\prod_{p} \mathscr{X}_{p}(\mathbf{s}, \mathbf{t})
$$

where

$$
\mathscr{X}_{p}(\mathbf{s}, \mathbf{t})=\lim _{T \rightarrow \infty} \frac{\# \mathscr{X}_{p, T}(\mathbf{s}, \mathbf{t})}{p^{n T}} .
$$

Using the fact that $\mathbf{t} \mid \mathbf{v}$ if and only if $\mathbf{t}^{[p]} \mid \mathbf{v}^{[p]}$ for all $p$, it follows from part (i) of Lemma 3.2 that

$$
\sum_{\substack{\mathbf{s}, \mathbf{t} \\ \mathbf{t} \mid \mathbf{v}}} \varpi(\mathbf{s}, \mathbf{t}) \frac{\mathfrak{S}_{\boldsymbol{\epsilon}} ; \mathbf{s}^{\mathbf{m}} \mathbf{w}}{\prod_{j=0}^{n} s_{j}}=\prod_{p}\left(\sum_{\substack{(\mathbf{s}, \mathbf{t})=\left(\mathbf{s}^{[p]}, \mathbf{t}^{[p]}\right) \\ \mathbf{t} \mid \mathbf{v}^{[p]}}} \varpi(\mathbf{s}, \mathbf{t}) \cdot \# \mathscr{X}_{p}(\mathbf{s}, \mathbf{t})\right) .
$$

On the other hand, on appealing to the inclusion-exclusion principle and the definition of $\varpi$, for any prime $p$ we return to (3.16) and see that

$$
\begin{aligned}
\mathscr{M}_{\boldsymbol{\epsilon}, T}(\mathbf{v}, p)= & \# \mathscr{X}_{p, T}(\mathbf{1}, \mathbf{1})-\# \bigcup_{\substack{(\mathbf{g} ; p ; I) \\
\mathbf{b}(\mathbf{g} ; p ; I) \mid \mathbf{v}^{[p]}}} \mathscr{X}_{p, T}(\mathbf{a}(\mathbf{g} ; p ; I), \mathbf{b}(\mathbf{g} ; p ; I)) \\
= & \# \mathscr{X}_{p, T}(\mathbf{1}, \mathbf{1})+\sum_{k=1}^{\infty}(-1)^{k} \sum_{\substack{\# \mathscr{R}=k \\
\mathscr{R}=\mathscr{R}(p) \\
\mathbf{b}(\mathscr{R}) \mid \mathbf{v}^{[p]}}} \# \mathscr{X}_{p, T}(\mathbf{a}(\mathscr{R}), \mathbf{b}(\mathscr{R})) \\
= & \sum_{\substack{(\mathbf{s}, \mathbf{t})=\left(\mathbf{S}^{[p]}, \mathbf{t}^{[p]}\right) \\
\mathbf{t} \mid \mathbf{v}^{[p]}}} \varpi(\mathbf{s}, \mathbf{t}) \cdot \# \mathscr{X}_{p, T}(\mathbf{s}, \mathbf{t}) .
\end{aligned}
$$

Dividing by $p^{n T}$ and taking the limit $T \rightarrow \infty$, we are now easily led to the proof of the claim (3.15).

\section{Thin sets: proof of Theorem 1.3}

Let $\Gamma=\sum_{j=0}^{n} \frac{1}{m_{j}}-1$, as in (2.2). In this section we assume that (1.3) holds and we let $\Omega \subset \mathbb{P}^{n-1}(\mathbb{Q})$ be a thin set. Theorem 1.3 is concerned with an upper bound for the quantity

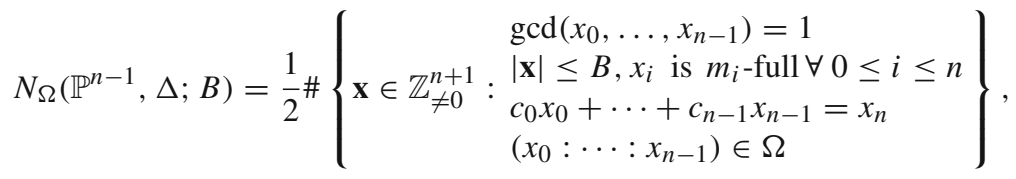


under the conditions on $\Omega$ that are stated in the theorem. Let us write $N_{\Omega}(B)=$ $N_{\Omega}\left(\mathbb{P}^{n-1}, \Delta ; B\right)$ to ease notation. All of the implied constants in this section are allowed to depend on the thin set $\Omega$.

We shall proceed by using information about the size of thin sets modulo $p$ on a set of primes $p$ of positive density. Our thin set $\Omega$ is contained in a finite union $\bigcup_{i=1}^{t} \Omega_{i}$ of thin subsets of type I and type II. We shall abuse notation and write $\Omega_{i}\left(\mathbb{F}_{p}\right)$ for the image of $\Omega_{i}$ in $\mathbb{P}^{n-1}\left(\mathbb{F}_{p}\right)$ under reduction modulo $p$. Similarly, we shall write $\widehat{\Omega}_{i}\left(\mathbb{F}_{p}\right)$ for the set of $\mathbb{F}_{p}$-points on the affine cone over this set of points.

Let $\Omega_{i} \subset \mathbb{P}^{n-1}(\mathbb{Q})$ be a thin subset of type I. Then it follows from the Lang-Weil estimates [9] that there exits $C_{1}>0$ such that

$$
\# \Omega_{i}\left(\mathbb{F}_{p}\right) \leq C_{1} p^{n-2},
$$

for every sufficiently large prime $p$. If $\Omega_{i} \subset \mathbb{P}^{n-1}(\mathbb{Q})$ is a thin subset of type II, then according to Serre [13, Thm. 3.6.2] there exists a constant $\kappa \in(0,1)$ such that

$$
\# \Omega_{i}\left(\mathbb{F}_{p}\right) \leq \kappa p^{n-1},
$$

for every sufficiently large prime $p \in \mathrm{P}_{\Omega_{i}}$, in the notation introduced before the statement of Theorem 1.3.

We take advantage of this information by noticing that

$$
N_{\Omega}(B) \leq \sum_{i=1}^{t} \#\left\{\mathbf{x} \in \mathbb{Z}_{\neq 0}^{n+1}: \begin{array}{l}
\operatorname{gcd}\left(x_{0}, \ldots, x_{n-1}\right)=1 \\
|\mathbf{x}| \leq B, x_{i} \text { is } m_{i}-\text { full } \forall 0 \leq i \leq n \\
c_{0} x_{0}+\cdots+c_{n-1} x_{n-1}=x_{n} \\
\left(x_{0}: \cdots: x_{n-1}\right) \bmod p \in \Omega_{i}\left(\mathbb{F}_{p}\right) \forall p \in \mathscr{S}_{i}
\end{array}\right\}
$$

for any finite subset of primes $\mathscr{S}_{i}$. We stipulate that $\min _{p \in \mathscr{S}_{i}} p$ is greater than some absolute constant depending only on $\prod_{i=0}^{n}\left|c_{i}\right| m_{i}$ and the thin subset $\Omega_{i}$. Let

$$
H_{i}=\prod_{p \in \mathscr{S}_{i}} p
$$

and put

$$
\Omega_{H_{i}}=\prod_{p \in \mathscr{S}_{i}} \widehat{\Omega}_{i}\left(\mathbb{F}_{p}\right) .
$$

Given $\mathbf{b}^{\prime}=\left(b_{0}, \ldots, b_{n-1}\right)$ we let

$$
b_{n}=c_{0} b_{0}+\cdots+c_{n-1} b_{n-1}
$$

and we put $\mathbf{b}=\left(b_{0}, \ldots, b_{n}\right)$. Appealing to (1.7) and putting $c_{n}=-1$, we deduce that $N_{\Omega}(B)$ is

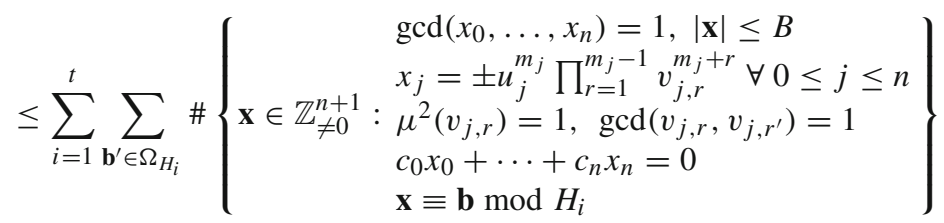

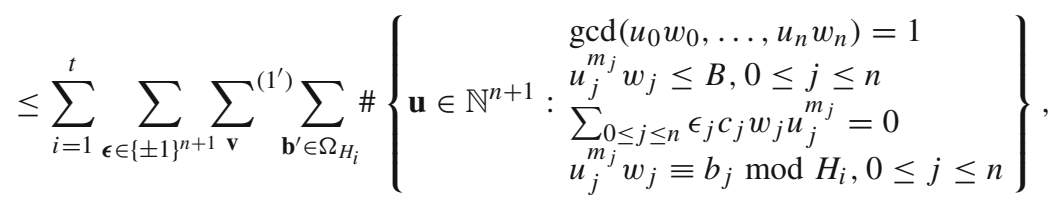


where $w_{j}=\prod_{r=1}^{m_{j}-1} v_{j, r}^{m_{j}+r}$ and $\sum_{\mathbf{v}}^{\left(1^{\prime}\right)}$ denotes a sum over $\mathbf{v}=\left(v_{0}, \ldots, v_{n}\right) \in \mathbb{N}^{n+1}$ satisfying $w_{j} \leq B$ and the coprimality conditions

$$
\operatorname{gcd}\left(v_{j, r}, v_{j, r^{\prime}}\right)=1, \quad \mu^{2}\left(v_{j, r}\right)=1, \quad \operatorname{gcd}\left(w_{0}, \ldots, w_{n}\right)=1 .
$$

(This should not be confused with the notation $\sum_{\mathbf{v}}^{(1)}$ in Sect. 3, in which the condition $\operatorname{gcd}\left(w_{0}, \ldots, w_{n}\right)=1$ does not appear.)

Let us define

$$
\Omega_{\mathbf{w} ; H_{i}}^{(i)}=\prod_{p \in \mathscr{S}_{i}} \Omega_{\mathbf{w} ; p}^{(i)},
$$

where

$$
\Omega_{\mathbf{w} ; p}^{(i)}=\left\{\mathbf{h} \in \mathbb{F}_{p}^{n+1} \backslash\{\mathbf{0}\}: \begin{array}{l}
h_{j}^{m_{j}} w_{j} \equiv b_{j} \bmod p \text { for } 0 \leq j \leq n \\
\text { for some } \mathbf{b}^{\prime} \in \widehat{\Omega}_{i}\left(\mathbb{F}_{p}\right)
\end{array}\right\} .
$$

In view of the coprimality conditions we are only interested in $\mathbf{b} \not \equiv \mathbf{0} \bmod p$ for all $p \in \mathscr{S}_{i}$. Thus, for each $p \in \mathscr{S}_{i}$ and $\mathbf{h} \in \Omega_{\mathbf{w} ; p}^{(i)}$ we have

$$
h_{j}^{m_{j}} w_{j} \not \equiv 0 \bmod p \quad \text { for some } j \in\{0, \ldots, n\} .
$$

With this notation, we may write

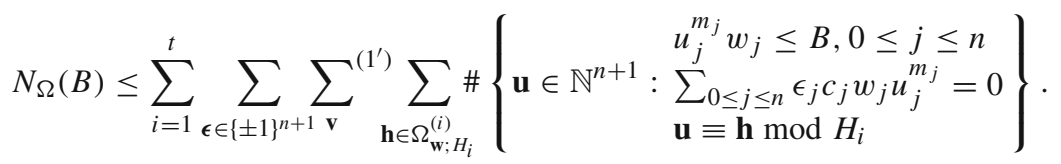

Note that $\# \Omega_{\mathbf{w} ; H_{i}}^{(i)} \leq H_{i}^{n+1}$. We now seek to apply Theorem 2.7 to the inner sum, much as in (3.13). Let $\eta>0$ be sufficiently small and assume that $\delta$ is chosen so that $(2 n+5) \delta=$ $\frac{1}{m_{*}\left(m_{*}+1\right)}-3 \eta$, where we have found it convenient to set $m_{*}=\max _{0 \leq j \leq n} m_{j}$. This is plainly satisfactory for (2.5). We take $\varepsilon=\eta$ in the statement of Theorem 2.7 and we assume that $H_{i}$ satisfies

$$
H_{i}^{n+1} \leq \min \left\{B^{\frac{\delta}{m_{*}\left(m_{*}+1\right)}-\varepsilon-\eta}, B^{\frac{1}{m_{*}\left(m_{*}+1\right)}-(2 n+5) \delta-\varepsilon-\eta}\right\}=B^{\eta},
$$

where the second equality is true provided that $\eta$ is small enough in terms of $m_{*}$ and $n$. Under this assumption it can be verified that the overall contribution from the error term in Theorem 2.7 is $O\left(B^{\Gamma-\eta}\right)$. It follows that

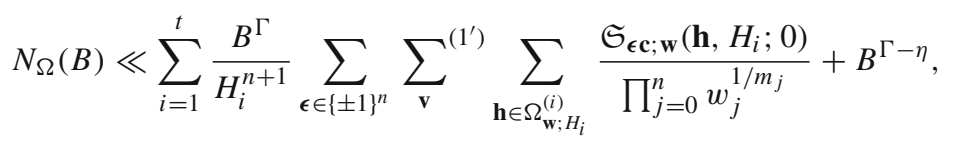

since $\mathfrak{J}_{\epsilon \mathbf{c}} \ll 1$.

Before proceeding with an analysis of the singular series, we first record some estimates for the size of $\Omega_{\mathbf{w} ; p}^{(i)}$, for any $i \in\{1, \ldots, t\}$.

Lemma 4.1 We have $\# \Omega_{\mathbf{w} ; p}^{(i)} \leq m_{*} p^{n}$ for any $p \in \mathscr{S}_{i}$.

Proof Suppose without loss of generality that $p \nmid w_{0}$ and let $h_{1}, \ldots, h_{n} \in \mathbb{F}_{p}$ be such that $h_{j}^{m_{j}} w_{j} \equiv b_{j}$ mod $p$ for $1 \leq j \leq n$, for some $\mathbf{b}^{\prime}$, where $b_{0}=c_{0}^{-1}\left(b_{n}-c_{1} b_{1}-\cdots-c_{n-1} b_{n-1}\right)$. Then there are at most $m_{0} \leq m_{*}$ choices for $h_{0}$. This confirms the lemma. 
Lemma 4.2 Assume that $p \in \mathscr{S}_{i}$ and $p \nmid w_{j}$ for $0 \leq j \leq n$. Then we have

$$
\# \Omega_{\mathbf{w} ; p}^{(i)} \leq \begin{cases}(p-1) \# \Omega_{i}\left(\mathbb{F}_{p}\right) & \text { if } p \in Q_{\mathbf{m}}, \\ m_{*}^{n}(p-1) \# \Omega_{i}\left(\mathbb{F}_{p}\right) & \text { otherwise }\end{cases}
$$

where $Q_{\mathbf{m}}$ is defined in (1.4).

Proof Let $z \in \Omega_{i}\left(\mathbb{F}_{p}\right)$. Either there are no points in $\Omega_{\mathbf{w} ; p}^{(i)}$ corresponding to $z$, or else we may assume that there exists $\mathbf{h} \in \mathbb{F}_{p}^{n+1} \backslash\{\mathbf{0}\}$ such that

$$
b_{j} \equiv h_{j}^{m_{j}} w_{j} \bmod p
$$

for $0 \leq j \leq n$, for some $\left(b_{0}, \ldots, b_{n}\right) \in \mathbb{F}_{p}^{n+1}$ such that $\left(b_{0}: \cdots: b_{n-1}\right)=z$, in which $b_{n}$ satisfies (4.3). Then the number of points in $\Omega_{\mathbf{w} ; p}^{(i)}$ associated to $z$ is at most the number of $a \in \mathbb{F}_{p}^{*}$ and $\mathbf{k} \in \mathbb{F}_{p}^{n+1}$ such that $a b_{j} \equiv k_{j}^{m_{j}} w_{j} \bmod p$ for $0 \leq j \leq n$. For fixed $a \in \mathbb{F}_{p}^{*}$, since $w_{j} \not \equiv 0 \bmod p$ for $0 \leq j \leq n$, it follows that the number of $\mathbf{k}$ is precisely the number of solutions to the set of congruences

$$
a h_{j}^{m_{j}} \equiv k_{j}^{m_{j}} \bmod p
$$

for $0 \leq j \leq n$.

If $b_{j}=0$ then it forces $h_{j}=0$, and so $k_{j}=0$. Suppose without loss of generality that $b_{j} \neq 0$ for $0 \leq j \leq R$ and $b_{R+1}=\cdots=b_{n}=0$. Let us fix a choice of a primitive element $g \in \mathbb{F}_{p}^{*}$ and put $a=g^{u}$, where $1 \leq u \leq p-1$. Then

$$
\begin{aligned}
& \#\left\{\mathbf{k} \in \mathbb{F}_{p}^{n+1}: a h_{j}^{m_{j}} \equiv k_{j}^{m_{j}} \bmod p \text { for } 0 \leq j \leq n\right\} \\
& =\#\left\{\left(x_{0}, \ldots, x_{R}\right) \in\left(\mathbb{F}_{p}^{*}\right)^{R+1}: x^{m_{j}}=a \text { for } 0 \leq j \leq R\right\} \\
& =\#\left\{\left(\ell_{0}, \ldots, \ell_{R}\right) \in(\mathbb{Z} /(p-1) \mathbb{Z})^{R+1}: \begin{array}{l}
m_{j} \ell_{j} \equiv u \bmod p-1 \\
\text { for } 0 \leq j \leq R
\end{array}\right\} \\
& \quad= \begin{cases}\prod_{j=0}^{R} \operatorname{gcd}\left(m_{j}, p-1\right) & \text { if } \operatorname{gcd}\left(m_{j}, p-1\right) \mid u \text { for } 0 \leq j \leq R, \\
0 & \text { otherwise. }\end{cases}
\end{aligned}
$$

In this way we see that

$$
\begin{aligned}
\sum_{a \in \mathbb{F}_{p}^{*}} \#\left\{\mathbf{k} \in \mathbb{F}_{p}^{n+1}: a h_{j}^{m_{j}} \equiv k_{j}^{m_{j}} \bmod p \text { for } 0 \leq j \leq n\right\} \\
=\frac{\prod_{0 \leq i \leq n} \operatorname{gcd}\left(m_{i}, p-1\right)}{\operatorname{lcm}\left(\operatorname{gcd}\left(m_{0}, p-1\right), \ldots, \operatorname{gcd}\left(m_{n}, p-1\right)\right)}(p-1) .
\end{aligned}
$$

The factor in front of $(p-1)$ is 1 when $p \in \mathbf{Q}_{\mathbf{m}}$ and at most $m_{*}^{n}$ in general. The statement of the lemma now follows.

We are now ready to analyse the singular series in (4.7). Let us put $c_{j}^{\prime}=\varepsilon_{j} c_{j}$ for indices $0 \leq j \leq n$. We recall from (2.11) that

$$
\mathfrak{S}_{\mathbf{c}^{\prime} ; \mathbf{w}}\left(\mathbf{h}, H_{i} ; 0\right)=\sum_{q=1}^{\infty} \frac{1}{q^{n+1}} \sum_{\substack{0 \leq a<q \\ \operatorname{gcd}(a, q)=1}} \prod_{j=0}^{n} \sum_{0 \leq k<q} e\left(\frac{a}{q} c_{j}^{\prime} w_{j}\left(H_{i} k+h_{j}\right)^{m_{j}}\right) .
$$


Put

$$
\mathscr{B}_{\mathbf{w}}\left(p^{t}\right)=\frac{1}{p^{t(n+1)}} \sum_{\substack{0 \leq a<p^{t} \\ \operatorname{gcd}\left(a, p^{t}\right)=1}} \prod_{j=0}^{n} \sum_{0 \leq k<p^{t}} e\left(\frac{a}{p^{t}} c_{j}^{\prime} w_{j}\left(H_{i} k+h_{j}\right)^{m_{j}}\right),
$$

so that

$$
\mathfrak{S}_{\mathbf{c}^{\prime} ; \mathbf{w}}\left(\mathbf{h}, H_{i} ; 0\right)=\prod_{p}\left(1+\sum_{t=1}^{\infty} \mathscr{B}_{\mathbf{w}}\left(p^{t}\right)\right)
$$

If $p \nmid H_{i}$ then

$$
1+\sum_{t=1}^{\infty} \mathscr{B}_{\mathbf{w}}\left(p^{t}\right)=1+\sum_{t=1}^{\infty} \frac{1}{p^{t(n+1)}} \sum_{\substack{0 \leq a<p^{t} \\ \operatorname{gcd}\left(a, p^{t}\right)=1}} \prod_{j=0}^{n} \sum_{0 \leq k<p^{t}} e\left(\frac{a}{p^{t}} c_{j}^{\prime} w_{j} k^{m_{j}}\right) .
$$

It now follows from (2.8) that

$$
\begin{aligned}
\prod_{p \nmid H_{i}}\left(1+\sum_{t=1}^{\infty} \mathscr{B}_{\mathbf{w}}\left(p^{t}\right)\right) & =\sum_{\substack{q=1 \\
\operatorname{gcd}\left(q, H_{i}\right)=1}}^{\infty} \frac{1}{q^{n+1}} \sum_{\substack{0 \leq a<q \\
\operatorname{gcd}(a, q)=1}} \prod_{j=0}^{n} \sum_{0 \leq k<q} e\left(\frac{a}{q} c_{j}^{\prime} w_{j} k^{m_{j}}\right) \\
& \ll \sum_{\substack{q=1 \\
\operatorname{gcd}\left(q, H_{i}\right)=1}}^{\infty} q^{1-\sum_{j=0}^{n} \frac{1}{m_{j}}+\varepsilon} \prod_{j=0}^{n} \operatorname{gcd}\left(q, w_{j}\right)^{\frac{1}{m_{j}}},
\end{aligned}
$$

for any $\varepsilon>0$. Moreover, in the usual way, for any prime $p$ we have

$$
1+\sum_{t=1}^{T} \mathscr{B}_{\mathbf{w}}\left(p^{t}\right)=p^{-n T} N\left(p^{T}\right)
$$

where

$$
N\left(p^{T}\right)=\#\left\{\mathbf{k} \bmod p^{T}: \sum_{j=0}^{n} c_{j}^{\prime} w_{j}\left(H_{i} k_{j}+h_{j}\right)^{m_{j}} \equiv 0 \bmod p^{T}\right\} .
$$

In order to deal with primes $p \mid H_{i}$, we require the following simple form of Hensel's lemma.

Lemma 4.3 Let $m, y, T \in \mathbb{N}$ and let $A, B \in \mathbb{Z}$. Assume that $p$ is prime such that $p \nmid A m y$ and $A y^{m}+B \equiv 0 \bmod p$. Then

$$
\#\left\{x \bmod p^{T}: A x^{m}+B \equiv 0 \bmod p^{T}, x \equiv y \bmod p\right\}=1 .
$$

Let $p \mid H_{i}$. Then $H_{i}=p H_{i}^{\prime}$ for some $H_{i}^{\prime} \in \mathbb{N}$ that is coprime to $p$. It readily follows that

$$
N\left(p^{T}\right)=p^{n+1} \#\left\{\mathbf{k} \bmod p^{T-1}: \sum_{j=0}^{n} c_{j}^{\prime} w_{j}\left(p k_{j}+h_{j}\right)^{m_{j}} \equiv 0 \bmod p^{T}\right\} .
$$

If $\mathbf{h} \bmod p$ is a solution to the congruence

$$
c_{0}^{\prime} w_{0} h_{0}^{m_{0}}+\cdots+c_{n}^{\prime} w_{n} h_{n}^{m_{n}} \equiv 0 \bmod p,
$$


then necessarily it is a non-singular solution by (4.5), since each prime $p \mid H_{i}$ is large enough that $p \nmid \prod_{j} c_{j}^{\prime} m_{j}$. Hence for $T>1$ it follows from Lemma 4.3 that $N\left(p^{T}\right)=$ $p^{n+1} p^{n(T-1)}=p^{n T+1}$. Bringing this together with (4.8) and (4.9) we conclude that

$$
\begin{aligned}
\mathfrak{S}_{\mathbf{c}^{\prime} ; \mathbf{w}}\left(\mathbf{h}, H_{i} ; 0\right) & =H_{i} \prod_{p \nmid H_{i}}\left(1+\sum_{t=1}^{\infty} \mathscr{B}_{\mathbf{w}}\left(p^{t}\right)\right) \\
& \ll H_{i} \sum_{\substack{q=1 \\
\operatorname{gcd}\left(q, H_{i}\right)=1}}^{\infty} q^{1-\sum_{j=0}^{n} \frac{1}{m_{j}}+\varepsilon} \prod_{j=0}^{n} \operatorname{gcd}\left(q, w_{j}\right)^{\frac{1}{m_{j}}} .
\end{aligned}
$$

Inserting this into (4.7), our work so far has shown that

$$
N_{\Omega}(B) \ll B^{\Gamma} \sum_{i=1}^{t} U\left(B, H_{i}\right)+B^{\Gamma-\eta},
$$

for any $\eta>0$, where

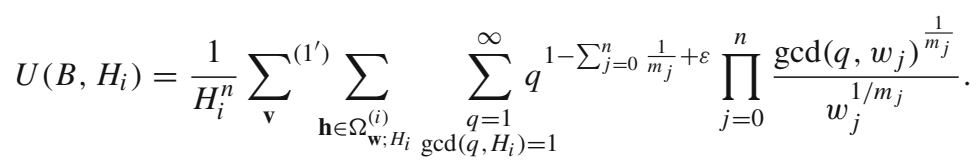

Let $1 \leq i \leq t$ and recall that $H_{i}=\prod_{p \in \mathscr{S}_{i}} p$. Appealing to (4.1), (4.2), together with Lemmas 4.1 and 4.2, we deduce that

$$
\# \Omega_{\mathbf{w} ; p}^{(i)} \leq \begin{cases}C_{1} m_{*}^{n} p^{n-1} & \text { if } p \nmid w_{j} \forall j \text { and } \Omega_{i} \text { is type I, } \\ \kappa p^{n} & \text { if } p \nmid w_{j} \forall j, \Omega_{i} \text { is type II and } p \in \mathrm{P}_{\Omega_{i}} \cap \mathrm{Q}_{\mathbf{m}}, \\ m_{*} p^{n} & \text { otherwise, }\end{cases}
$$

for some $\kappa \in(0,1)$.

Suppose first that $\Omega_{i}$ is type I and let $\omega\left(H_{i}\right)=\# \mathscr{S}_{i}$. Then

$$
\begin{aligned}
\frac{\# \Omega_{\mathbf{w} ; H_{i}}^{(i)}}{H_{i}^{n}} \leq \prod_{\substack{p \mid H_{i} \\
p \nmid w_{0} \ldots w_{n}}} \frac{C_{1} m_{*}^{n}}{p} \prod_{\substack{p\left|H_{i} \\
p\right| w_{0} \ldots w_{n}}} m_{*} & =\frac{\left(C_{1} m_{*}^{n}\right)^{\omega\left(H_{i}\right)}}{H_{i}} \prod_{p \mid \operatorname{gcd}\left(H_{i}, w_{0} \ldots w_{n}\right)} \frac{p m_{*}}{C_{1} m_{*}^{n}} \\
& \ll H_{i}^{-1+\varepsilon} \operatorname{gcd}\left(H_{i}, w_{0} \ldots w_{n}\right),
\end{aligned}
$$

for any $\varepsilon>0$. But then it follows that

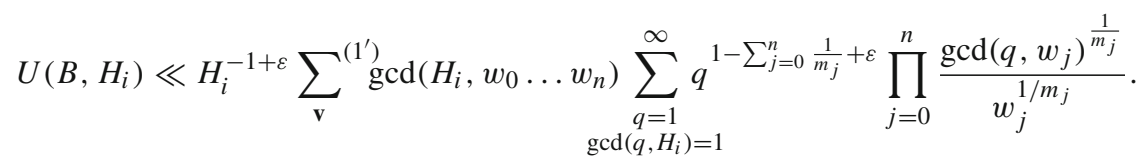

Observe that $\operatorname{val}_{p}\left(w_{j}\right) \geq m_{j}+1$ whenever $p \mid w_{j}$. In this way we can see that

$$
\operatorname{val}_{p}\left(\frac{\operatorname{gcd}\left(H_{i}, w_{0} \ldots w_{n}\right)}{\prod_{j=0}^{n} w_{j}^{1 / m_{j}}}\right) \leq \frac{-1}{m_{*}}
$$


for every prime $p$ such that $p \mid H_{i}$ and $p \mid w_{0} \ldots w_{n}$. Thus, on removing common factors of $w_{j}$ with $H_{i}$, one easily concludes that

$$
U\left(B, H_{i}\right) \leq H_{i}^{-1+\varepsilon} g\left(H_{i}\right) \sum_{\substack{w_{j} \leq B \\ \operatorname{gcd}\left(w_{j}, H_{i}\right)=1 \\(4.4) \text { holds }}} \sum_{\substack{q=1 \\ \operatorname{gcd}\left(q, H_{i}\right)=1}}^{\infty} q^{1-\sum_{j=0}^{n} \frac{1}{m_{j}}+\varepsilon} \prod_{j=0}^{n} \frac{\operatorname{gcd}\left(q, w_{j}\right)^{\frac{1}{m_{j}}}}{w_{j}^{1 / m_{j}}},
$$

where $g\left(H_{i}\right)=\prod_{p \mid H_{i}}\left(1+O\left(p^{-1 / m_{*}}\right)\right)$. It is an elementary exercise to show that

$$
g\left(H_{i}\right) \leq \exp \left(\frac{C_{2}\left(\log H_{i}\right)^{1-1 / m_{*}}}{\log \log H_{i}}\right),
$$

for an absolute constant $C_{2}>0$. Taking $g\left(H_{i}\right) \ll H_{i}^{\varepsilon}$ for any $\varepsilon>0$, it now follows from (2.10) and (3.10) that $U\left(B, H_{i}\right) \ll_{\varepsilon} H_{i}^{-1+2 \varepsilon}$. Once inserted into (4.10) and choosing $\mathscr{S}_{i}$ in such a way that $H_{i}$ is a small enough power of $B$ for (4.6), this shows that thin subsets of type I make a satisfactory overall contribution.

Suppose next that $\Omega_{i}$ is type II. We may assume that $p \geq m_{*} / \kappa$ for each $p \mid H_{i}$. Then

$$
\begin{aligned}
\frac{\# \Omega_{\mathbf{w} ; H_{i}}^{(i)}}{H_{i}^{n}} \leq \prod_{\substack{p \mid H_{i} \\
p \nmid w_{0} \ldots w_{n} \\
p \in \mathrm{P}_{\Omega_{i}} \cap \mathrm{Q}_{\mathbf{m}}}} \kappa \prod_{\substack{p\left|H_{i} \\
p\right| w_{0} \ldots w_{n}}} m_{*} & \leq \prod_{\substack{p \mid H_{i} \\
p \in \mathrm{P}_{\Omega_{i}} \cap \mathrm{Q}_{\mathbf{m}}}} \kappa \prod_{p \mid \operatorname{gcd}\left(H_{i}, w_{0} \ldots w_{n}\right)} \frac{m_{*}}{\kappa} \\
& \leq \operatorname{gcd}\left(H_{i}, w_{0} \ldots w_{n}\right) \prod_{\substack{p \mid H_{i} \\
p \in \mathrm{P}_{\Omega_{i}} \cap \mathrm{Q}_{\mathbf{m}}}} \kappa .
\end{aligned}
$$

We choose $\mathscr{S}_{i}$ to be set of primes $1 \ll p \leq \log B / \log \log B$ drawn from the set $\mathrm{P}_{\Omega_{i}} \cap \mathrm{Q}_{\mathbf{m}}$. In particular $H_{i}$ satisfies (4.6). Moreover, it follows from our assumption (1.5) that this set of primes has positive lower density $\varrho$, say. But then

$$
\prod_{\substack{p \mid H_{i} \\ p \in \mathrm{P}_{\Omega_{i}} \cap \mathbf{Q}_{\mathbf{m}}}} \kappa=\prod_{p \mid H_{i}} \kappa \leq\left(\frac{1}{\kappa}\right)^{-\frac{\varrho \log B}{(\log \log B)^{2}}} .
$$

Feeding this into the argument that we have just given yields

$$
U\left(B, H_{i}\right) \ll \exp \left(\frac{C_{2}\left(\log H_{i}\right)^{1-1 / m_{*}}}{\log \log H_{i}}-\frac{\log (1 / \kappa) \varrho \log B}{(\log \log B)^{2}}\right) \ll \frac{1}{(\log B)^{100}},
$$

from which it follows that the thin subsets of type II make a satisfactory overall contribution to (4.10) under the assumption (1.5). This completes the proof of Theorem 1.3.

Acknowledgements While working on this paper the authors were both supported by EPSRC grant $\mathrm{EP} / \mathrm{P} 026710 / 1$, and the second author received additional support from the NWO Veni Grant 016.Veni.192 .047. Thanks are due to Marta Pieropan, Arne Smeets and Sho Tanimoto for useful conversations related to this topic, and to the anonymous referee for numerous helpful suggestions.

Open Access This article is licensed under a Creative Commons Attribution 4.0 International License, which permits use, sharing, adaptation, distribution and reproduction in any medium or format, as long as you give appropriate credit to the original author(s) and the source, provide a link to the Creative Commons licence, and indicate if changes were made. The images or other third party material in this article are included in the article's Creative Commons licence, unless indicated otherwise in a credit line to the material. If material is 
not included in the article's Creative Commons licence and your intended use is not permitted by statutory regulation or exceeds the permitted use, you will need to obtain permission directly from the copyright holder. To view a copy of this licence, visit http://creativecommons.org/licenses/by/4.0/.

\section{References}

1. Bourgain, J., Demeter, C., Guth, L.: Proof of the main conjecture in Vinogradov's mean value theorem for degrees higher than three. Ann. Math. 184, 633-682 (2016)

2. Browning, T.D., Van Valckenborgh, K.: Sums of three squareful numbers. Experiment. Math. 21, 204-211 (2012)

3. Brüdern, J.: Sums of squares and higher powers. J. Lond. Math. Soc. 35, 233-243 (1987)

4. Campana, F.: Fibres multiples sur les surfaces: aspects geométriques, hyperboliques et arithmétiques. Manuscr. Math. 117, 429-461 (2005)

5. Cohen, S.D.: The distribution of Galois groups and Hilbert's irreducibility theorem. Proc. Lond. Math. Soc. 3, 227-250 (1981)

6. Davenport, H.: Analytic Methods for Diophantine Equations and Diophantine Inequalities, 2nd edn. Cambridge University Press, Cambridge (2005)

7. Franke, J., Manin, Y.I., Tschinkel, Y.: Rational points of bounded height on Fano varieties. Invent. Math. 95, 421-435 (1989)

8. Heath-Brown, D.R.: A new form of the circle method and its application to quadratic forms. J. Reine Angew. Math. 481, 149-206 (1996)

9. Lang, S., Weil, A.: Number of points of varieties in finite fields. Am. J. Math. 76, 819-827 (1954)

10. Pieropan, M., Schindler, D.: Hyperbola method on toric varieties (2020). arXiv:2001.09815

11. Pieropan, M., Smeets, A., Tanimoto, S., Várilly-Alvarado, A.: Campana points of bounded height on vector group compactifications. Proc. Lond. Math. Soc

12. Serre, J.-P.: Lectures on the Mordell-Weil Theorem. Aspects of Mathematics, 3rd edn. Friedr. Vieweg \& Sohn, Braunschweig (1997)

13. Serre, J.-P.: Topics in Galois theory. 2nd edition. Research Notes in Mathematics 1, A. K. Peters, Ltd., Wellesley, MA (2008)

14. Van Valckenborgh, K.: Squareful numbers in hyperplanes. Algebra Number Theory 6, 1019-1041 (2012)

15. Vaughan, R. C.: The Hardy-Littlewood Method, 2nd ed., Cambridge Tracts in Math. 125, Cambridge Univ. Press, Cambridge (1997)

16. Wooley, T.D.: Vinogradov's mean value theorem via efficient congruencing. Ann. Math. 175, 1575-1627 (2012)

17. Wooley, T.D.: The asymptotic formula in Waring's problem. Int. Math. Res. Not. 2012(7), 1485-1504 (2012)

18. Wooley, T.D.: The cubic case of the main conjecture in Vinogradov's mean value theorem. Adv. Math. 294, 532-561 (2016)

19. Wooley, T.D.: Nested efficient congruencing and relatives of Vinogradov's mean value theorem. Proc. Lond. Math. Soc. 118, 942-1016 (2019)

Publisher's Note Springer Nature remains neutral with regard to jurisdictional claims in published maps and institutional affiliations. 